Portland State University

PDXScholar

Winter 3-12-2015

\title{
Design of a Hardware Platform for GPS-Based Orientation Sensing
}

Daniel Eugene Kirkpatrick

Portland State University

Follow this and additional works at: https://pdxscholar.library.pdx.edu/open_access_etds

Part of the Electrical and Computer Engineering Commons, and the Navigation, Guidance, Control and Dynamics Commons

Let us know how access to this document benefits you.

\section{Recommended Citation}

Kirkpatrick, Daniel Eugene, "Design of a Hardware Platform for GPS-Based Orientation Sensing" (2015). Dissertations and Theses. Paper 2197.

https://doi.org/10.15760/etd.2194

This Thesis is brought to you for free and open access. It has been accepted for inclusion in Dissertations and Theses by an authorized administrator of PDXScholar. Please contact us if we can make this document more accessible: pdxscholar@pdx.edu. 
Design of a Hardware Platform for GPS-Based Orientation Sensing

by

Daniel Eugene Kirkpatrick

A thesis submitted in partial fulfillment of the requirements for the degree of

\author{
Master of Science \\ in \\ Electrical and Computer Engineering
}

Thesis Committee:

James McNames, Chair

Mark Faust

Roy Kravitz

Portland State University

2015 
(C) 2015 Daniel Eugene Kirkpatrick 


\begin{abstract}
Unmanned aerial vehicles (UAV's) have recently gained popularity in military, civil service, agriculture, commercial, and hobby use. This is due in part to their affordability, which comes from advances in component technology. That technology includes microelectromechanical systems (MEMS) for inertial sensing, microprocessor technology for sequential algorithm processing, field programmable gate arrays (FPGA's) for parallel data processing, camera technology, global navigation satellite systems (GNSS's) for navigation, and battery technology such as the high energy density of lithium polymer batteries.

Despite the success of the technology to date, there remains development before UAV's should be flying alongside manned aircraft or over populated areas. One concern is that UAV electronics are not as safe, reliable or robust as manned-aircraft electronics because UAV's are not certified by the FAA. Another concern for UAV operation is with control algorithms and sensors, particularly in the estimation of the aircraft state, which is the position, velocity, and orientation of the aircraft. Some problems, such as numerical stability of a control algorithm or flight in windy and turbulent conditions have only been solved for certain conditions of wind, weather, or maneuvers. Outside those conditions, the actual orientation of a flying craft can mislead to the control system, and the control system may not be able to recover without a crash. When pilots fly manned aircraft in instrument meteorological conditions, or conditions of limited visibility of the ground, terrain, and obstacles, the pilot must fly in a manner which avoids abrupt maneuvers which could disturb accuracy of the aircraft's instruments. In a UAV without a pilot, there
\end{abstract}


is a need to estimate the position and orientation of a UAV in an absolute manner unambiguous relative to the Earth. The position and orientation estimate must not depend on carefully controlled flight paths, but instead the estimate must be robust in the presence of UAV flight dynamics.

This thesis describes the design, implementation, and evaluation of a hardware platform for GPS based orientation sensing research. In this work, we considered a receiver with three or four RF sections, each connected to an antenna in a triangular or tetrahedral pyramid constellation. Specific requirements for the receiver hardware and functionality were created. Circuitry was designed to meet the requirements using commercial off-the-shelf (COTS) radio frequency (RF) modules, a mid-sized microcontroller, an FPGA, and other supporting components. A printed circuit board (PCB) was designed, fabricated, assembled, and tested. A GPS baseband processor was designed and coded in Verilog hardware description language. The design was synthesized and loaded to the FPGA, and the microcontroller was programmed to track satellites.

With the hardware platform implemented, live satellite signals were found and tracked, and experiments were performed to explore the validity of GPS based orientation sensing using short antenna baselines. The platform successfully allows the user to develop correlator designs and explore carrier phase based orientation measurement using only software/Verilog modifications. Initial results of carrier phase based orientation sensing are promising, but the presence of multipath signal interference shows room for improvement to the baseband processing code. 


\section{Dedication}

This thesis is dedicated to my father, who tirelessly answered every question asked about how the world works; to my mother, who supported and encouraged me on my lifelong journey through education; to my wife, who held the kids at bay while I write this; and to everyone that let me explain the elegance and genius of GPS. 


\section{Acknowledgments}

The origin of this project was part of a Portland State University, Maseeh College of Engineering and Computer Science (MCECS) "Beta project" (formerly known as an innovation project), which was awarded funds in 2011. The goal of that project was to advance technology toward creating a UAV which could locate lost people in remote mountain or forest terrain by flying search patterns and using infrared sensitive cameras. I would like to thank the MCECS Beta project board for their financial and technical support of the UAV project which, in part, funded this thesis project.

Further, I would like to thank Rob Gaskell and Jeremy Booth for their participation in the Beta project, for their review of my schematic and HDL work on this thesis, and for listening to my explanations of how GPS works. I likely learned as much by explaining how GPS works to them as I did reading the subject for myself.

Finally, I want to thank my thesis adviser, Dr James McNames, and committee members, Professors Mark Faust and Roy Kravitz, for their encouragement and willingness to accept only my best work. 


\section{Table of Contents}

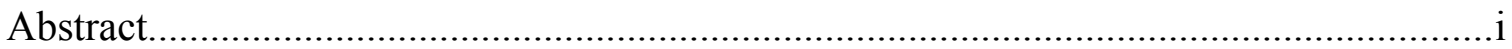

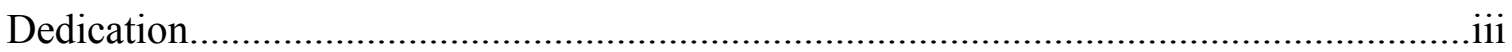

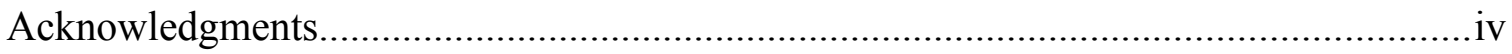

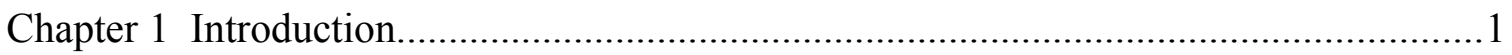

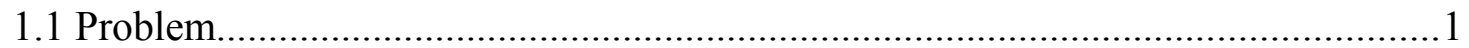

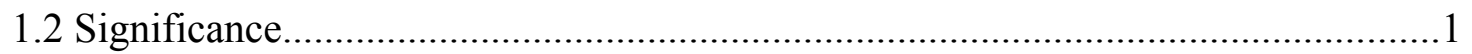

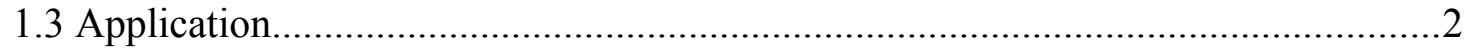

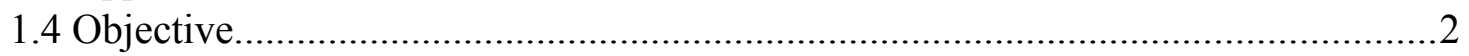

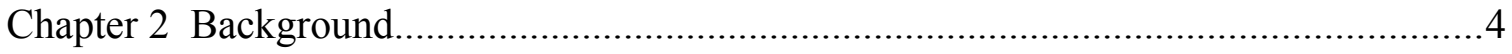

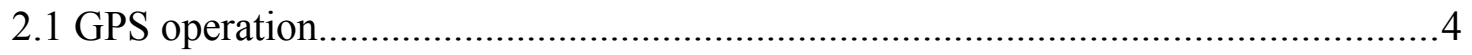

2.2 GPS L1 signal structure..............................................................................

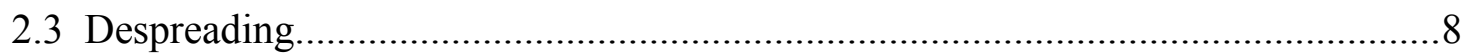

2.4 GPS applied to absolute attitude....................................................................

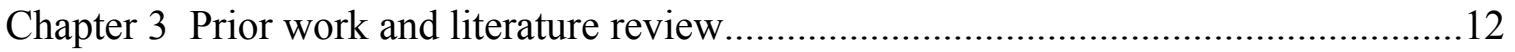

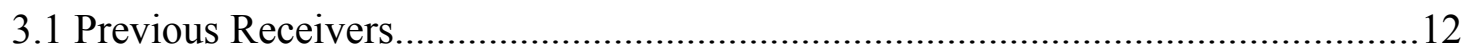

3.2 Previous Orientation Estimates....................................................................... 13

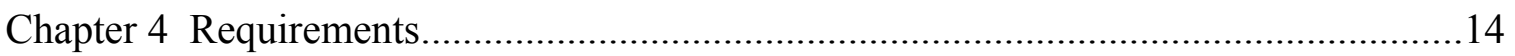

4.1 Functional requirements - high level functionality......................................... 15

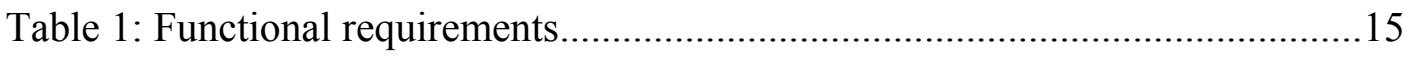

4.2 Environmental requirements - operating conditions........................................ 15

Table 2: Environmental requirements............................................................. 15

4.3 Mechanical requirements - vibration tolerance and physical mounting...............16

Table 3: Mechanical requirements............................................................. 16

4.4 Electrical requirements - supply voltage, current, and electrical interface............16

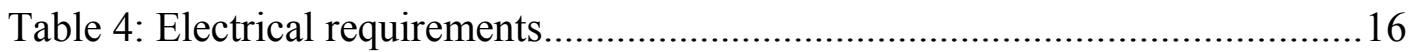

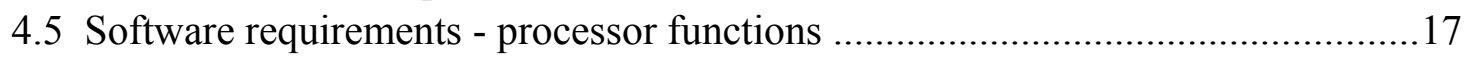

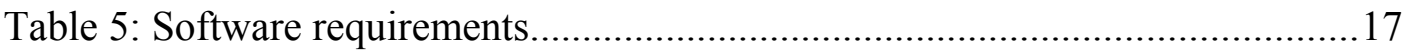

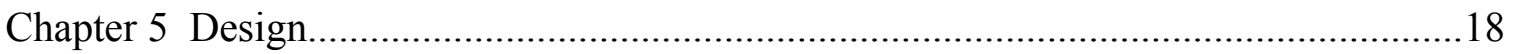

5.1 Hardware design........................................................................................ 18

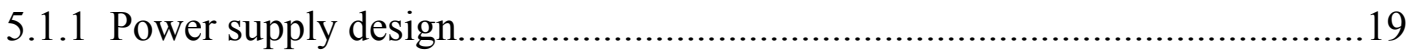

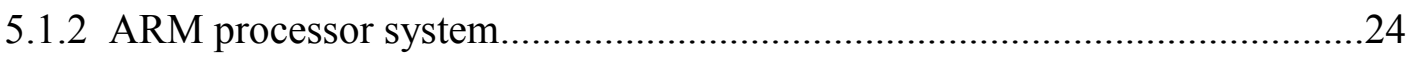

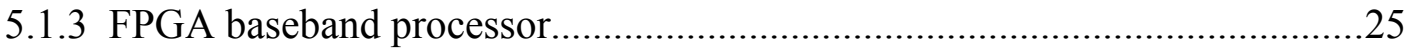

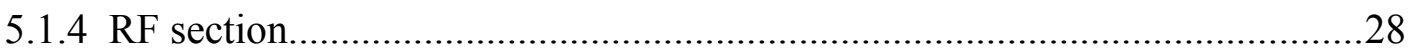

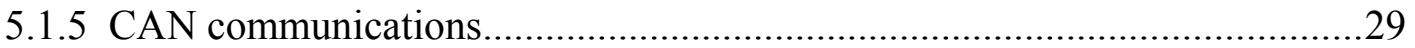

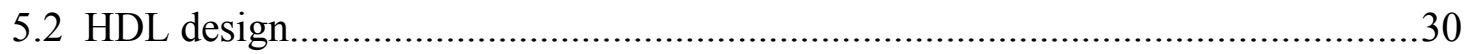

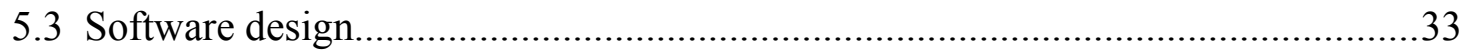

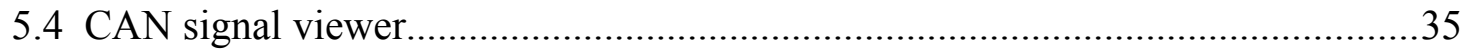

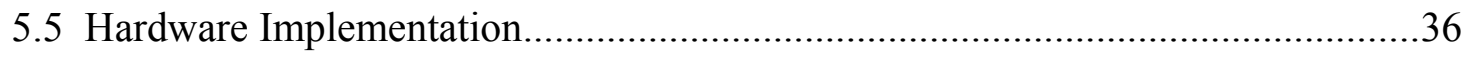

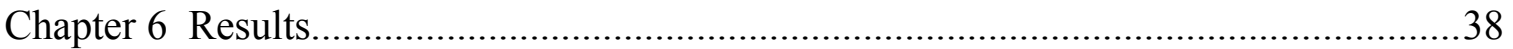




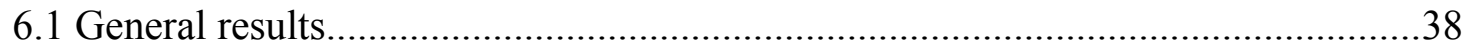

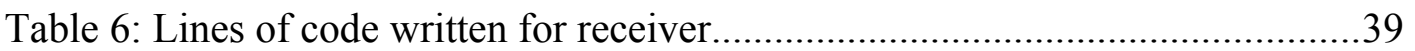

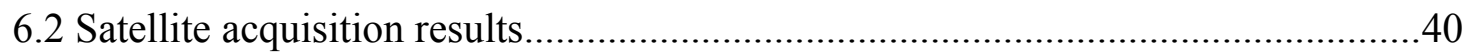

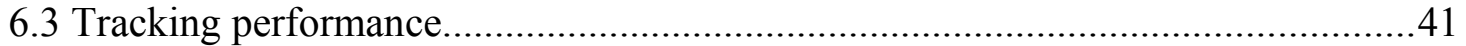

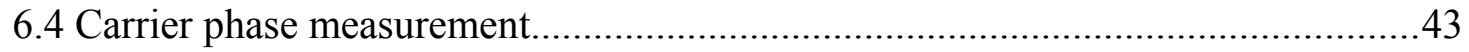

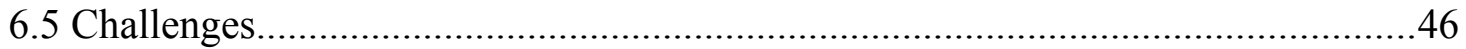

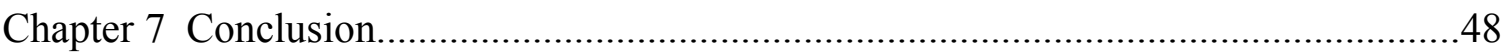

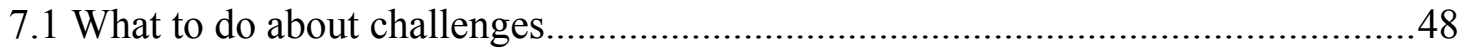

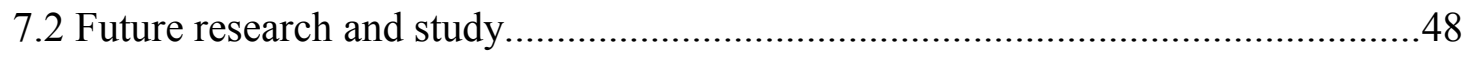

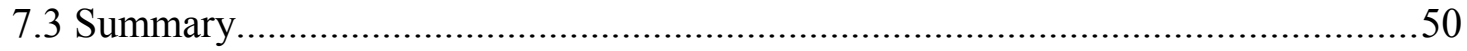

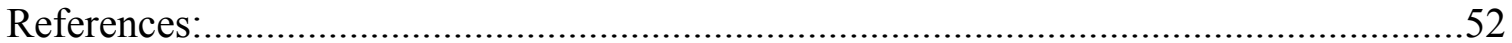




\section{List of Tables}

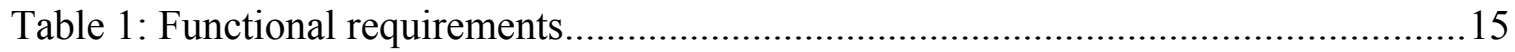

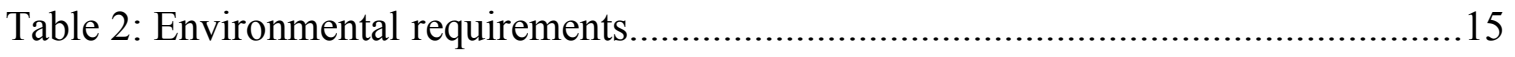

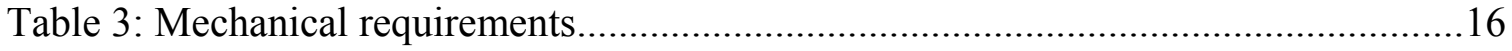

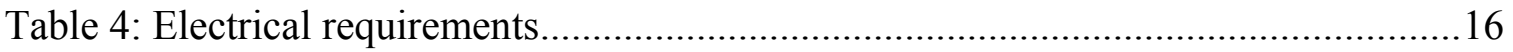

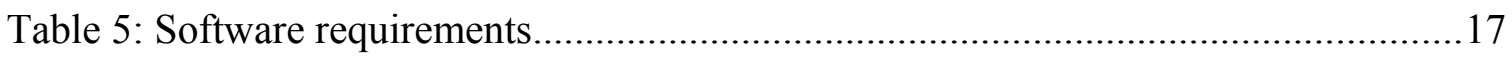

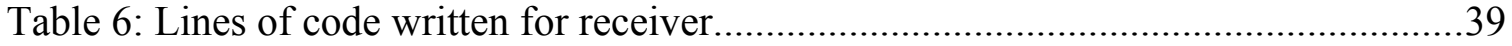




\section{List of Figures}

Figure 2.1: Signal spectrum of GPS L1 PRN 38 showing power spectral density vs. frequency offset from carrier.

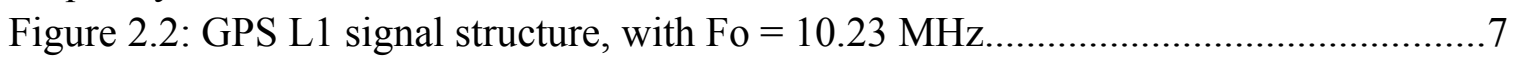

Figure 2.3: GPS radio wave-front arriving at antenna constellation................................9

Figure 2.4: GPS antenna array attached to UAV body frame....................................... 10

Figure 5.1: Receiver block diagram showing major sub-systems................................ 19

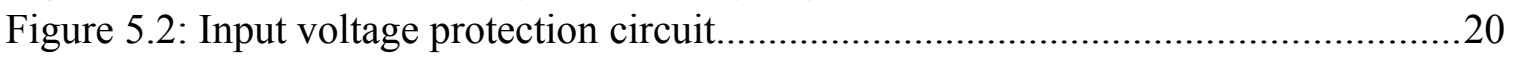

Figure 5.3: Switching power supply, $1.2 \mathrm{~V}$....................................................................21

Figure 5.4: Linear voltage regulator circuits............................................................22

Figure 5.5: Receiver power distribution for all supply voltages..................................23

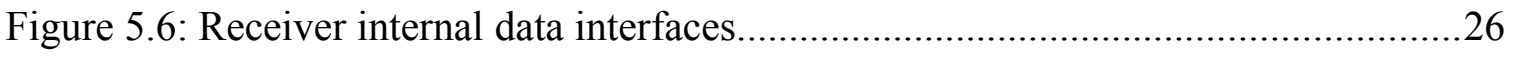

Figure 5.7: FPGA - ARM read cycle timing diagram...............................................2

Figure 5.8: GPS501 RF module frequency plan...........................................................29

Figure 5.9: Correlator block diagram with tracking loops............................................. 30

Figure 5.10: Antenna orientation measured from carrier phase ..................................32

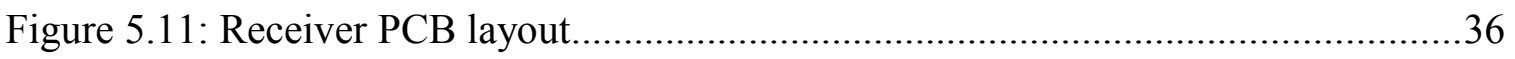

Figure 5.12: PCB assembly with components in place..................................................37

Figure 6.1: CAN signal view of satellite acquisition and DLL/FLL tracking..................41

Figure 6.2: CAN signal view of correlators 0 and 1 in PLL track state.........................42

Figure 6.3: Test platform for antenna rotation estimates............................................43

Figure 6.4: CAN signal view graph of I integration during rotation................................44

Figure 6.5: Multipath signal interference arriving at antenna array..............................46 


\section{Chapter 1 Introduction}

\subsection{Problem}

In an unmanned aerial vehicle (UAV) control system, estimating the aircraft state, such as the position, velocity, and orientation relative to the Earth is a challenging problem. Often, the state estimate will diverge some amount from the actual state during flight. The state estimate can become unstable if it diverges too far from the actual state during aggressive flight maneuvers [1]. Part of the state, specifically the orientation of the aircraft relative to the Earth, can be estimated by starting with a known orientation and integrating angular velocity to maintain the estimate. Small errors and noise in angular velocity measurements are also integrated over time. If uncorrected, they become large errors in the estimate. There are no absolute reference sensors to correct an error in orientation, so the orientation error must be corrected indirectly using acceleration measurements (including gravity), magnetic heading, GPS position, and GPS velocity. This is effective, but it is computationally intensive and can lead to unstable state estimates for highly dynamic maneuvers [1]. Though a proof is outside the scope of this thesis, if an absolute orientation reference were available, it is the author's contention that aircraft orientation estimates could be corrected in a more robust manner, and also with less computational power.

\subsection{Significance}

This thesis describes hardware used to explore a GPS based absolute orientation sensor for UAV's. Larger aircraft, such as those found in general aviation (GA), can 
make use of the longer wingspan and larger airframe to separate the GPS antennas farther apart, which would produce better orientation measurement accuracy and lower measurement noise [4][11]. Although any antenna baseline could be used with this hardware, this work is tested using a $4.76 \mathrm{~cm}$ baseline. The shorter baseline is better suited to small UAV airframes where antenna size and separation is limited. More importantly, the short antenna baseline reduces computational requirements by eliminating integer wavelength ambiguities inherent to GPS based orientation with long baselines. The orientation solution can be augmented with a low cost inertial navigation sensor (INS) to smooth the noise from the short baseline orientation estimate. Using an absolute orientation reference, the reduced computational load in the control system allows a lighter and lower-power control system, which enables the UAV to be built smaller and allows more energy to be provided to the aircraft's other systems.

\subsection{Application}

In this thesis, an autonomous or partially autonomous UAV is the intended application. However, instead of a commercial UAV, the intended test aircraft for this work is a radio controlled (RC) helicopter, and the hardware designed and built for this thesis is an experimental research platform. The research platform mounted to an RC helicopter allows low cost experimentation, while still allowing actual flight data collection which mimics commercial UAV flight.

\subsection{Objective}

This thesis describes the design and implementation of a hardware platform for research on GPS-based orientation sensing. The platform combines RF sections, power 
supplies, an ARM microprocessor, and an FPGA for GPS signal processing experiments. Unlike previous research GPS platforms [2][3], the hardware described enables a user to process GPS signals from up to four antennas. The platform allows RF sections to be interchanged, so that different RF sections can be evaluated side by side with the same baseband processor. With three or four homogeneous RF sections sharing the same clock, a baseband processor can be created to research GPS based orientation sensing, tightly coupled GPS INS integration, or any part of GPS baseband processing such as the following: discriminators, accumulators, timing components, oscillators, master/slave correlators, etc. 


\section{Chapter 2 Background}

\subsection{GPS operation}

The Global Positioning System (GPS) is one type of Global Navigation Satellite System (GNSS), and is the satellite system used in this project. GNSS positioning systems use space-based satellite signals to provide accurate position, velocity and time information for a receiver on or above the surface of the Earth. While the U.S.-built GPS system is the most widely used to date, other GNSS constellations include GLONASS (Russia), Galileo (European), and BeiDou (China).

A GPS receiver locates the receiver's position, or more precisely the position of the receiver antenna, by computing the time of flight of radio frequency signals traveling to the receiver from the satellites in the constellation. This is accomplished by tracking (receiving signals from) a number of GPS satellites that are within radio range of the receiver. The receiver computes the position of each satellite tracked using data streamed from the satellite. The satellite positions are computed in a coordinate system fixed to the center of and rotating with the Earth, called Earth-Centered Earth-Fixed (ECEF) coordinates. The satellite data also contains information regarding when the data is transmitted. By recording when the data is received, an approximation of the time required for the signal to travel from the satellite to the receiver is computed. Given that radio signals travel at the speed of light, an approximate range (distance) from the satellite to the receiver is determined. Although applying this range from three satellites is sufficient to determine the position of the receiver, an accurate position would not be

possible without an extremely accurate clock located at the receiver to measure the exact 
time that the signals are received. To compensate for the inaccuracy and drift of the receiver clock, one additional satellite signal must be tracked to solve for the clock error and compensate its effects. This makes intuitive sense, in that the receiver is using four inputs (ranges to four satellites) to solve for four unknowns; the clock error, $\mathrm{X}, \mathrm{Y}$, and Z ECEF position of the receiver.

Additional satellite signals and ranges (more than the requisite four), are used to improve the accuracy of the receiver position solution in the presence of noise in the satellite range approximation. Similar to using many data points to improve measurement of a linear regression model, added satellites improve accuracy in a receiver's position, velocity and time estimates.

\subsection{GPS L1 signal structure}

GPS operates in the IEEE "L" radio band. For this project, the satellite signals tracked will be in the GPS L1 frequency, which is centered at $1575.42 \mathrm{MHz}$. The GPS L1 signal is a spread spectrum signal, meaning that the signal has a center frequency of 1575.42 MHz, but the signal power is intentionally spread over a wide frequency range. The spectrum of frequencies that contain power from the GPS L1 signal is wider than +-50 MHz, but a typical L1 GPS receiver will limit received RF bandwidth to between +-2 MHz and +-10 MHz. Figure 2.1 shows the signal spectrum of an L1 GPS PRN signal. 


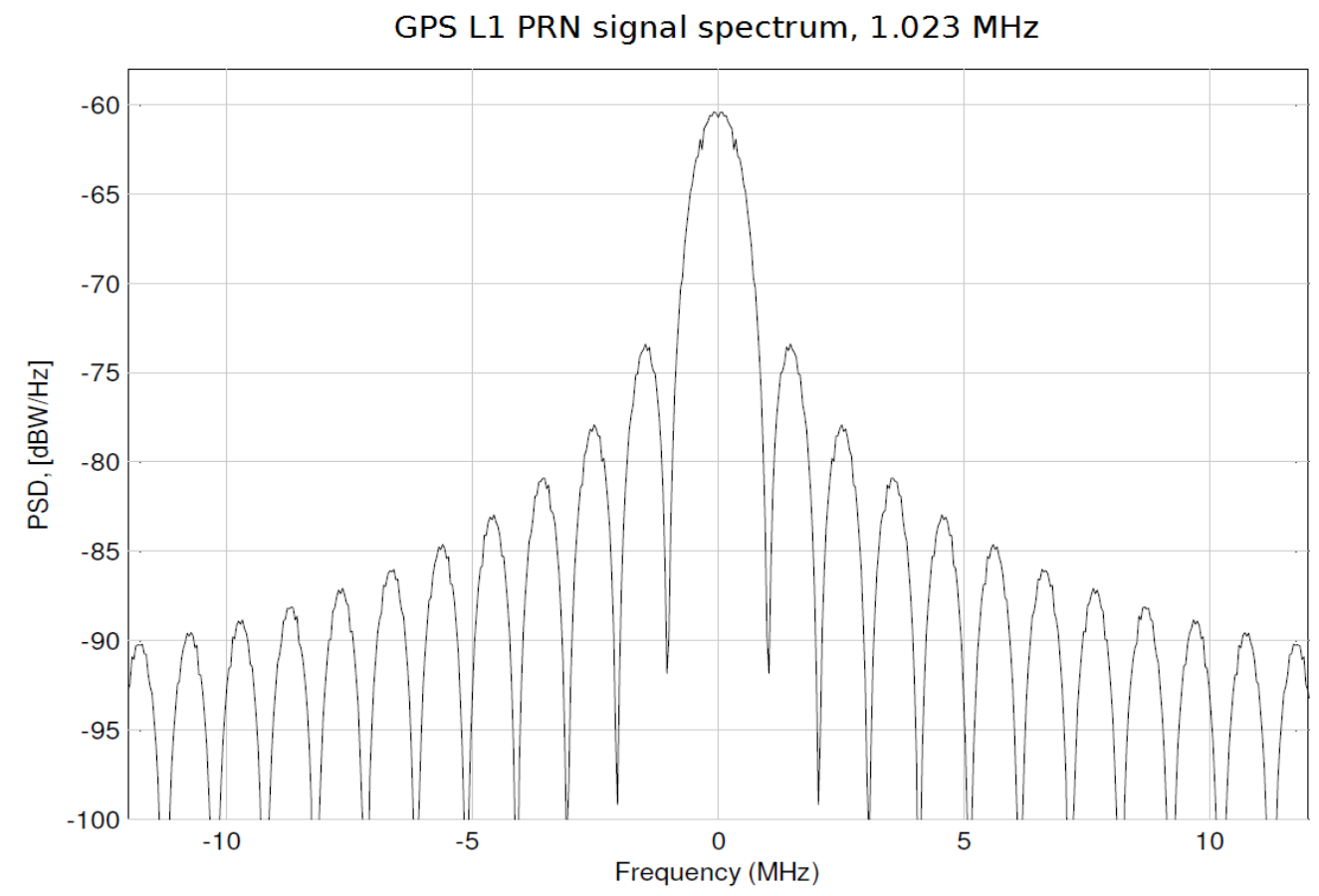

Figure 2.1: Signal spectrum of GPS L1 PRN 38 showing power spectral density vs. frequency offset from carrier.

Using a bandwidth of less than $2 \mathrm{MHz}$ decreases the signal to noise ratio (SNR) of the receiver because more of the desired signal is outside the RF filter bandwidth. Using a bandwidth wider than $20 \mathrm{MHz}$ will also decrease the receiver SNR because more noise power will enter the receiver with little added contribution to signal power. A designer optimizes the receiver by selecting the RF bandwidth versus other design choices such as correlator chip spacing, required accuracy, power consumption, and cost.

The L1 GPS signal power is spread over a range of frequencies by modulating the carrier using binary phase shift keying (BPSK) modulation with a pseudo random number (PRN) sequence, combined with the navigation data the satellite intends to transmit. A PRN is a sequence of binary values that has statistical properties similar to a 
random number. A GPS L1 PRN is a repeating sequence of 1023 data bits, called "chips". Figure 2.2 shows the idealized time series values of the L1 carrier, PRN, and the combined, modulated signal as transmitted by a GPS satellite.
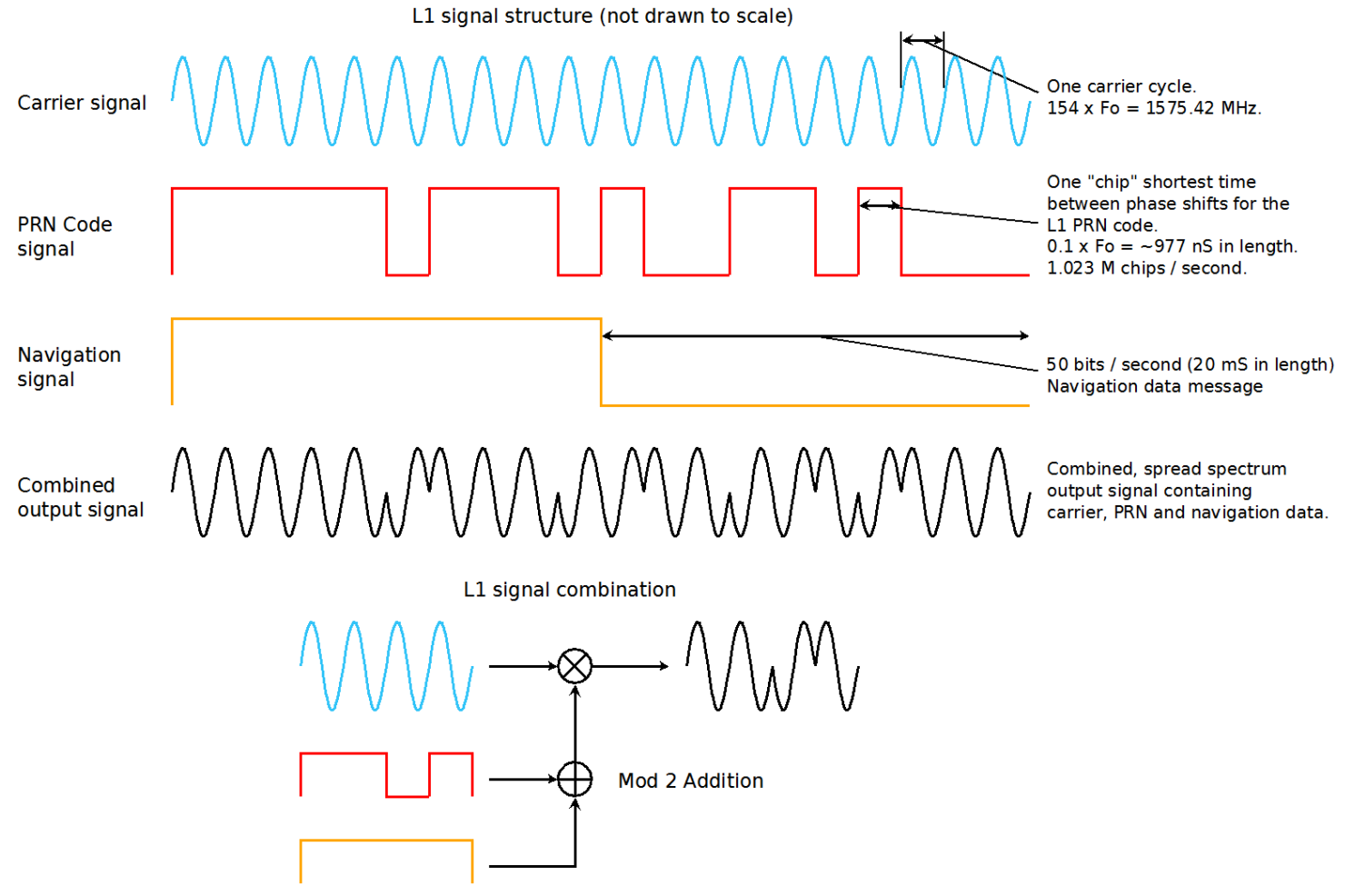

Figure 2.2: GPS L1 signal structure, with Fo $=10.23 \mathrm{MHz}$

Unlike a true random number, PRN's are sequences of numbers that repeat. Because PRN's are repeating sequences, they can be duplicated in a receiver and used to despread a spread spectrum signal. Actual navigation and PRN codes require a finite slew rate, and therefore the carrier phase transitions are not as clean as shown in Figure 2.2.

GPS PRN's belong to a special class of PRN's called gold codes. Gold code PRN's differ from each other in a way which minimizes the likelihood that one PRN would be mistaken for another. Mathematically, that is to say that two different gold codes have a low cross correlation. For the GPS L1 signals, a gold code PRN sequence can be 
generated from a pair of shift registers and several XOR gates. Each satellite transmits an L1 carrier BPSK modulated both by a gold code PRN unique to that satellite, and by a binary stream of data.

GPS satellites are in orbits around the Earth. Because the satellites and possibly the receiver are moving, the relative motion between the satellite and the receiver changes the L1 carrier and PRN modulation frequencies by as much as $+-5 \mathrm{kHz}$ and $+-3 \mathrm{~Hz}$, respectively, due to the Doppler effect [5]. The receiver must be capable of searching for and tracking the carrier and PRN over this frequency range.

\subsection{Despreading}

A GPS L1 satellite signal is received at very low power because the signal power is spread over tens of megahertz. At a received signal power level of around -125dBm [5], the power of a GPS signal is below that of background noise received at the antenna from space. The spread spectrum GPS signal must be de-spread before it has sufficient signal to noise ratio to be tracked. Despreading concentrates the power of a GPS signal onto a single frequency so that the information carried on the signal can be extracted.

Within the baseband processor, a GPS receiver despreads the GPS L1 signal by creating a local copy of the known PRN code for a given satellite, and mixing (multiplying) the local PRN by the incoming signal. This mixing of the local PRN reverses the PRN modulation of the carrier in the satellite, leaving the $1575.42 \mathrm{MHz}$ carrier and the BPSK satellite data with a higher signal to noise ratio than the received spread spectrum signal. 


\subsection{GPS applied to absolute attitude}

The intent of this project is to build a hardware platform which measures the orientation of the receiver relative to the ECEF reference frame. The position of the receiver antenna and the satellites are assumed known from the receiver position solution which may come from this receiver or from a separate GPS receiver. Orientation is measured by comparing the phase relationship between GPS satellite signals as they reach each antenna in a constellation of receiver antennas as shown in Figure 2.3.

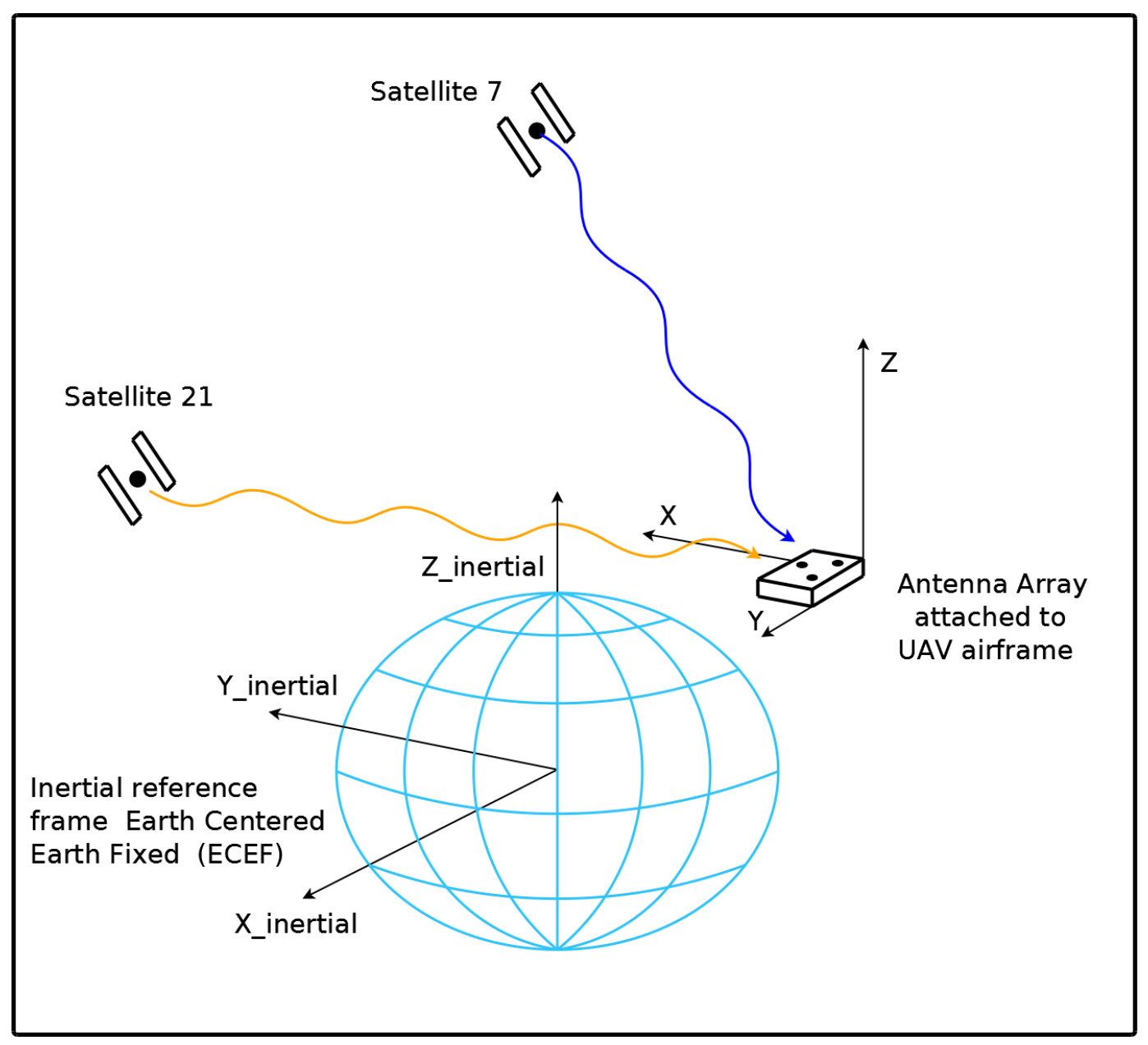

Figure 2.3: GPS radio wave-front arriving at antenna constellation 
The antennas in the constellation have a fixed spacing between them of $14^{\text {th }}$ of the GPS carrier frequency wavelength $(\lambda / 4$, in Figure 2.4$)$, which is approximately $4.76 \mathrm{~cm}$. This spacing is used to support small antenna constellations on UAV's, and is also used because it contains no integer wavelength ambiguities when measuring angles to the satellites. More specifically, GPS satellite signals are compared by measuring the difference in carrier phase of the satellite signal from different receiver antennas, as shown in Figure 2.4.

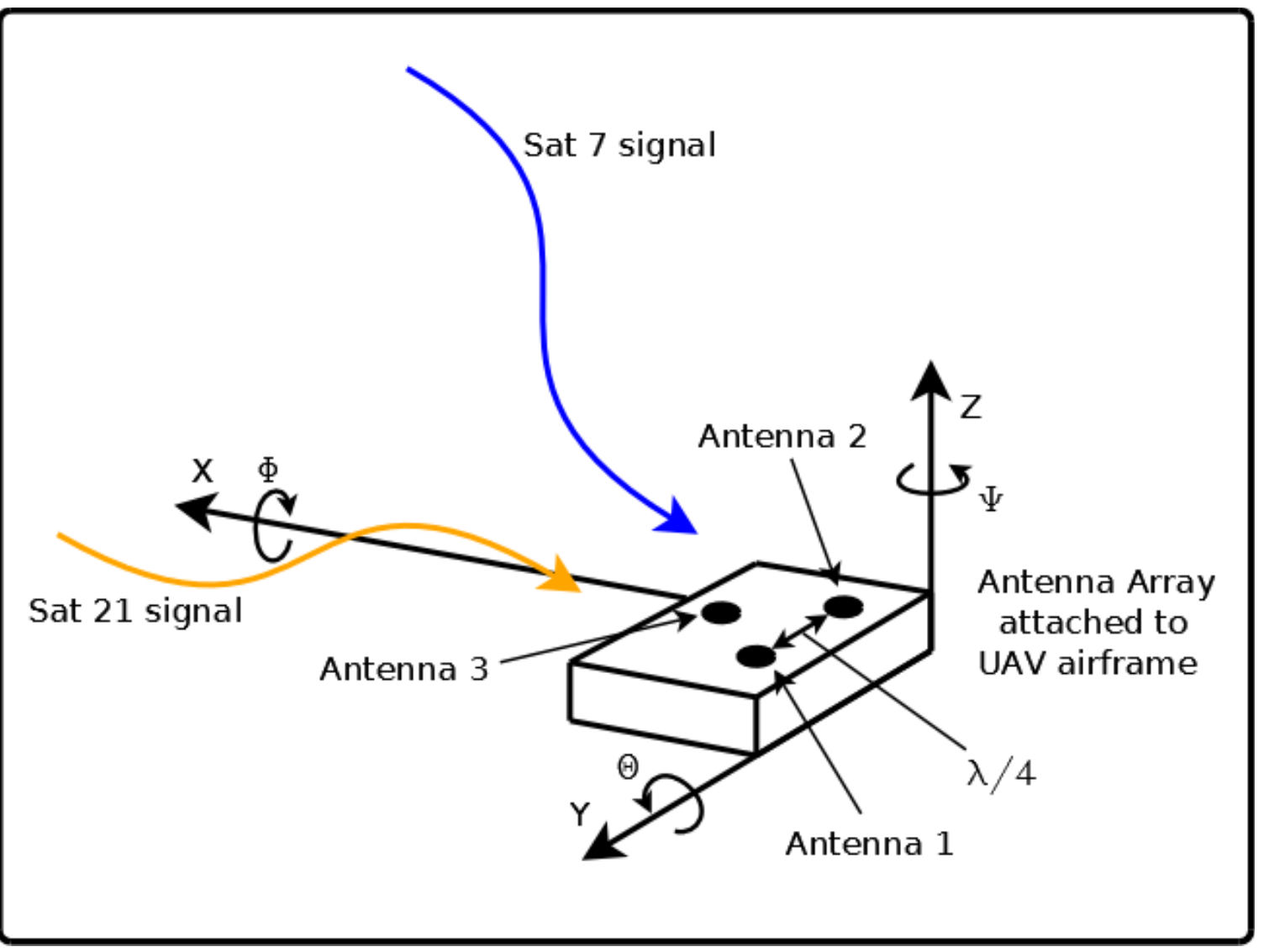

Figure 2.4: GPS antenna array attached to UAV body frame 
The carrier phase measurement gives the direction of the satellite relative to the antenna constellation. Given the known ECEF antenna position and satellite positions, the ECEF orientation of the antenna constellation can be determined.

Although it is not demonstrated in this thesis, it is assumed that additional satellite direction measurements improve the accuracy of the orientation estimate, similar to the improved receiver position obtained from additional satellite range measurements. This, along with best methods for combining the data, are the intended research this hardware platform enables. 


\section{Chapter 3 Prior work and literature review}

\subsection{Previous Receivers}

This project was inspired in part by two previous projects. The first was GPL-GPS, which was a masters thesis project by Andrew Greenberg at Portland State University [2]. In the GPL-GPS project, existing GPS receiver hardware consisting of an RF front end and a baseband processor was used, and an open source GPS receiver software base was ported to the hardware to create the first portable open source GPS receiver.

Around the same time, another open-hardware open-baseband-processor project called Namuru [3] was created by Peter Mumford, Kevin Parkinson and Andrew Dempster as part of a project at the University of New South Wales (UNSW) in New Zealand. The Namuru receiver used an existing RF chipset with an FPGA baseband processor and supporting software to create a GPS receiver. The GPL-GPS project used a single antenna to track the receiver position, while the Namuru project provided two antennas for tracking either L1 and L2 signals, or two L1 signals.

The extension created in this thesis is the application of multiple RF front ends attached to multiple receiver antennas, with the intent of determining the absolute orientation of the antenna set relative to the Earth. Neither the GPL-GPS or Namuru project provided the three or four RF inputs needed to obtain a full GPS based orientation solution. 


\subsection{Previous Orientation Estimates}

GPS based orientation has been studied in the past. With flight tests involving general aviation (GA) aircraft, the resolution and accuracy are reported to have been less than one degree error; however, the results were obtained with long baselines (distances between the antennas) of more than 15 meters [4]. In the formative works on potential applications of GPS technology (reference [11]), GPS based orientation sensing is introduced with justification for theoretical accuracy. For GA aircraft, long antenna baselines are simple to accommodate given the wingspan and length of the fuselage. For a UAV, long baselines are not practical because small UAV's give no structure to mount an antenna on a long baseline. Long baselines also span many of the GPS carrier wavelengths. Only fractional wavelengths can be directly measured, so a particular GPS carrier phase measured at the antennas may map to many different orientations. Long baseline receivers expend significant effort to solve the integer wavelength ambiguity problem, which is eliminated in this design. 


\section{Chapter 4 Requirements}

As an experimental platform for GPS based orientation, the receiver requires a combination of features that provide immediate functionality, as well as features that make the project extensible. For example, the receiver must provide interface to an antenna constellation for primary GPS orientation functionality; but the receiver must also be portable for eventual flight testing. Another extensibility requirement is an oversize power supply current capacity. Providing extra power supply capability allows full use of the FPGA for logic with little risk of over loading the power supplies in low density air. The following requirements for this receiver project are grouped into five categories. 


\subsection{Functional requirements - high level functionality}

Table 1: Functional requirements

\begin{tabular}{|l|l|l|}
\hline Number & Receiver requirement & Justification \\
\hline 4.1 .1 & $\begin{array}{l}\text { Shall use GPS L1 satellite signals to } \\
\text { determine orientation of antenna } \\
\text { constellation relative to satellite } \\
\text { constellation (met) }\end{array}$ & $\begin{array}{l}\text { The GPS L1 constellation is the intended constellation. } \\
\text { Other constellations are at various levels of } \\
\text { completion, but as of the date of implementation of this } \\
\text { project, GPS L1 is the only viable alternative. }\end{array}$ \\
\hline 4.1 .2 & $\begin{array}{l}\text { Shall receive GPS L1 satellite signals } \\
\text { from a four antenna constellation, } \\
\text { simultaneously (met) }\end{array}$ & $\begin{array}{l}\text { A minimum of three antennas and two satellites are } \\
\text { necessary for attitude determination, but a fourth } \\
\text { antenna could aid in conditioning attitude solutions } \\
\text { from poor satellite geometry [11] }\end{array}$ \\
\hline 4.1 .3 & $\begin{array}{l}\text { Shall interface (share data) with other } \\
\text { embedded control systems (met) }\end{array}$ & $\begin{array}{l}\text { The system must provide some means of } \\
\text { communicating its status with the outside world, and } \\
\text { considering the eventual intended UAV application, } \\
\text { the communication must be with other systems which } \\
\text { use, record, or transmit the data. }\end{array}$ \\
\hline 4.1 .4 & $\begin{array}{l}\text { Shall be capable of flight test on an RC } \\
\text { helicopter (met) }\end{array}$ & $\begin{array}{l}\text { With the intended UAV application, flight test via RC } \\
\text { helicopter or quad copter is necessary to keep costs } \\
\text { within a reasonable range }\end{array}$ \\
\hline 4.1 .5 & $\begin{array}{l}\text { Shall provide flexibility to experiment } \\
\text { with GPS RF modules, baseband } \\
\text { processors, and correlator designs } \\
\text { (met) }\end{array}$ & $\begin{array}{l}\text { As a research device (as opposed to a finished, } \\
\text { marketable product), there must be capability to vary } \\
\text { the design in order to try different options for RF } \\
\text { designs, signal processing, algorithms, etc. }\end{array}$ \\
\hline
\end{tabular}

\subsection{Environmental requirements - operating conditions}

Table 2: Environmental requirements

\begin{tabular}{|l|l|l|}
\hline Number & Receiver requirement & Justification \\
\hline 4.2 .1 & $\begin{array}{l}\text { Shall operate in humidity levels } \\
\text { between 5\% to 95\% relative humidity } \\
\text { (will be met with a PCB enclosure } \\
\text { during flight test) }\end{array}$ & $\begin{array}{l}\text { In a UAV operating environment, temperature, } \\
\text { humidity, and air pressure vary greatly with seasons, } \\
\text { weather and operating altitude. Operating electronics in } \\
\text { high temperatures or in low density air may require } \\
\text { derating of air cooled components and system design. } \\
\text { Operation in high humidity requires isolation of } \\
\text { components inside enclosures or conformal coatings. }\end{array}$ \\
\hline 4.2 .2 & $\begin{array}{l}\text { Shall operate at altitudes from sea } \\
\text { level to 15,000 ft MSL (met by design) }\end{array}$ & $\begin{array}{l}\text { Shall operate in temperatures from 0 - } \\
70 \text { degrees C (met by design) }\end{array}$ \\
\hline 4.2 .3 & \multicolumn{2}{|l}{} \\
\hline
\end{tabular}




\subsection{Mechanical requirements - vibration tolerance and physical mounting}

Table 3: Mechanical requirements

\begin{tabular}{|l|l|l|}
\hline Number & Receiver requirement & Justification \\
\hline 4.3 .1 & $\begin{array}{l}\text { Shall provide mounting points for } \\
\text { main PCB less than } 10 \mathrm{~cm} \text { apart (met) }\end{array}$ & $\begin{array}{l}\text { PCB mounts are needed to provide secure attachment } \\
\text { to a UAV platform. Screw type connectors or soldered } \\
\text { wire pigtails provide a solid mechanical connection. A } \\
\text { small helicopter may see vibrations in the range of 0 - } \\
300 \mathrm{~Hz}[1] .\end{array}$ \\
\hline 4.3 .2 & $\begin{array}{l}\text { Shall provide mounting points for } \\
\text { attached RF PCB's (met) }\end{array}$ & $\begin{array}{l}\text { Shall provide through hole soldered or } \\
\text { screw terminal connections for } \\
\text { external wiring (met) }\end{array}$ \\
\hline 4.3 .3
\end{tabular}

\subsection{Electrical requirements - supply voltage, current, and electrical interface}

Table 4: Electrical requirements

\begin{tabular}{|c|c|c|}
\hline Number & Receiver requirement & Justification \\
\hline 4.4 .1 & $\begin{array}{l}\text { Shall operate from a single power } \\
\text { supply (met) }\end{array}$ & $\begin{array}{l}\text { RC helicopter power supplies operate from } 9 \mathrm{~V} \\
\text { (drained } 3 \text { cell lithium) to over } 25 \mathrm{~V} \text { (connected to half } \\
\text { of a } 44 \mathrm{~V} 12 \text { cell lithium system). }\end{array}$ \\
\hline 4.4 .2 & $\begin{array}{l}\text { Shall operate at voltages from } 9 \mathrm{~V} \text { to } \\
36 \mathrm{~V} \text { (met) }\end{array}$ & \\
\hline 4.4 .3 & $\begin{array}{l}\text { Shall draw less than } 2.0 \mathrm{~A} \text { current } \\
\text { (met) }\end{array}$ & \\
\hline 4.4 .4 & $\begin{array}{l}\text { Shall provide up to } 2.0 \mathrm{~A} \text { current } \\
\text { capacity to FPGA } 1.2 \mathrm{~V} \text { and } 2.5 \mathrm{~V} \\
\text { rails (met) }\end{array}$ & \\
\hline 4.4 .5 & $\begin{array}{l}\text { Shall provide antenna supply voltage } \\
\text { of } 5.0 \mathrm{~V} \text { (met) }\end{array}$ & $\begin{array}{l}\text { GPS antennas are powered by the receiver. Typical } \\
\text { specification is } 5 \mathrm{~V} \text { at } 30 \mathrm{~mA} \text {. }\end{array}$ \\
\hline 4.4 .6 & $\begin{array}{l}\text { Shall provide antenna supply current } \\
\text { up to } 100 \mathrm{~mA} \text { per antenna (met by } \\
\text { design) }\end{array}$ & \\
\hline 4.4 .7 & $\begin{array}{l}\text { Shall provide external communication } \\
\text { via CAN bus (met) }\end{array}$ & $\begin{array}{l}\text { A low latency, noise tolerant communication system } \\
\text { must be used to transfer data from the receiver. }\end{array}$ \\
\hline 4.4 .8 & $\begin{array}{l}\text { Shall use a baseband processor } \\
\text { capable of supporting at least six } \\
\text { baseband correlators from each of the } \\
\text { four antennas (met, }<50 \% \text { FPGA use) }\end{array}$ & $\begin{array}{l}\text { Six satellites (four is absolute minimum) from each } \\
\text { antenna. }\end{array}$ \\
\hline
\end{tabular}




\begin{tabular}{|l|l|l|}
\hline 4.4 .9 & $\begin{array}{l}\text { Shall use processor capable of } \\
\text { supporting correlator tracking loops as } \\
\text { well as low latency data transfer (met) }\end{array}$ & \\
\hline 4.4 .10 & $\begin{array}{l}\text { Shall provide debugging and } \\
\text { programming via separate JTAG } \\
\text { connectors for each programmable } \\
\text { device (met) }\end{array}$ & \\
\hline 4.4 .11 & $\begin{array}{l}\text { Shall synchronize all RF sections to } \\
\text { one reference clock (met) }\end{array}$ & \\
\hline 4.4 .12 & $\begin{array}{l}\text { Shall provide noise isolated power } \\
\text { supplies to digital and RF sections } \\
\text { (met) }\end{array}$ & \\
\hline
\end{tabular}

\subsection{Software requirements - processor functions}

Table 5: Software requirements

\begin{tabular}{|l|l|l|}
\hline Number & Receiver requirement & Justification \\
\hline 4.5 .1 & $\begin{array}{l}\text { Shall provide interrupt latency less } \\
\text { than 500 ns (met with processor } \\
\text { selection) }\end{array}$ & $\begin{array}{l}\text { It is expected that interrupts from the 24 correlators } \\
\text { will require servicing at most every 1 ms, so at 500 ns, } \\
\text { latency will account for less than 2\% of that time. }\end{array}$ \\
\hline 4.5 .2 & $\begin{array}{l}\text { Shall provide loop filters for satellite } \\
\text { tracking loops (met with SW design) }\end{array}$ & $\begin{array}{l}\text { One topic of study for GPS research is tight coupling } \\
\text { between GPS and inertial sensors. To accommodate } \\
\text { tight coupling and ease of experiments, the correlator } \\
\text { loop filters are located in the ARM processor. }\end{array}$ \\
\hline $\begin{array}{l}\text { 4.5.3 } \\
\text { analysis and performance monitoring }\end{array}$ & $\begin{array}{l}\text { Shall provide live data stream for } \\
\text { Without data from the receiver, there is no way to tell } \\
\text { what is going on inside the receiver. A live data stream } \\
\text { from the processor is used to debug both hardware and } \\
\text { software, as well as provide data to systems which use } \\
\text { the orientation data. }\end{array}$ \\
\hline
\end{tabular}




\section{Chapter 5 Design}

\subsection{Hardware design}

Due to the $0^{\circ} \mathrm{C}-70^{\circ} \mathrm{C}$ temperature requirements, commercial grade or better components were used throughout this design. To meet temperature requirements for air cooled devices up to the $15,000 \mathrm{ft}$ MSL limit, no special design adjustments are required because ambient cooling air temperature decreases with altitude faster than loss of free convective cooling caused by reduced air density [12].

The project requirements for flight test on an RC helicopter and flexibility to experiment drive the design of a printed circuit board (PCB) which has power supplies, processors, an FPGA, and communications on one PCB. The complexity of an RF circuit design with sufficient noise performance for GPS is outside the scope of this computer engineering project, so a COTS solution, the GPS Creations GPS501 GPS L1 RF downconverter is used.

Figure 5.1 shows the basic block diagram for the receiver design. 


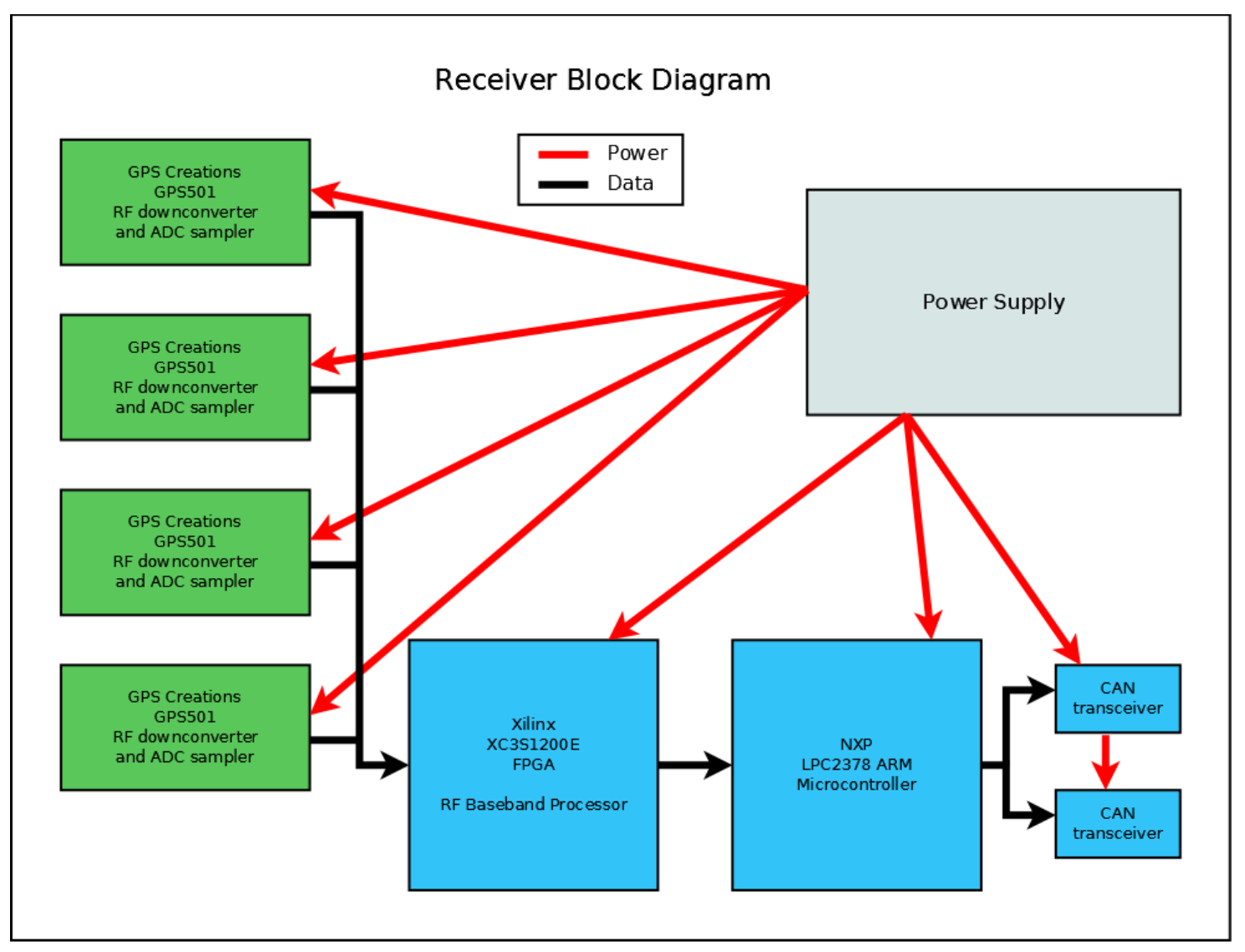

Figure 5.1: Receiver block diagram showing major sub-systems

\subsubsection{Power supply design}

The design goal is to provide sufficient power to RF, FPGA, processor, and communications transceivers while also providing separate sources for noise producing digital devices and noise susceptible analog or RF devices. Supplies were chosen to provide significantly more current capability than required. In this way, changes to the design during testing and development, changes to power consumption in the FPGA, and changes to RF power consumption would not likely drive changes to the power supply.

The supply system accepts input voltages between $9 \mathrm{~V}$ and $36 \mathrm{~V}$ to meet power input requirements, and protects itself from damage caused by improper input 
connections. The power supply input protection circuit consists of a $40 \mathrm{~V}$ Zener clamp diode, capacitor, and fuse. Together, they provide protection from power supply voltages over $40 \mathrm{~V}$ and from connection to reverse voltages. Figure 5.2 shows the input protection circuit.

\section{Input Protection}

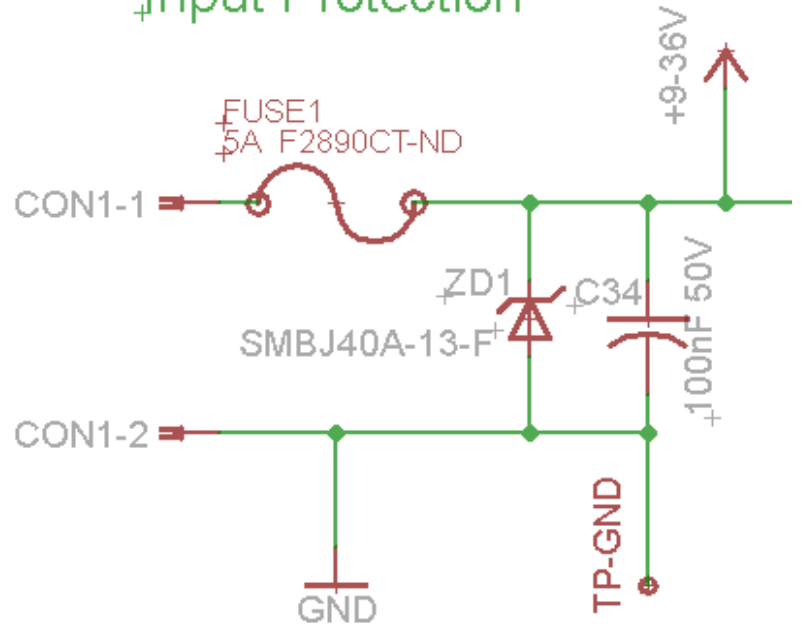

Figure 5.2: Input voltage protection circuit

The protection circuit is designed to cause high current flow in the diode during an over-voltage or reverse-voltage condition. This high current will exceed the $5 \mathrm{~A}$ fuse rating, and the fuse will open the input supply circuit before any damage is done to downstream components which must remain below $40 \mathrm{~V}$. For input voltages below $36 \mathrm{~V}$, the fuse and diode components do not contribute significantly to the circuit operation.

Three Texas Instruments LMZ14203 switching power supplies (SPS) compose the primary voltage regulators. The LMZ14203 is a highly integrated supply, with internal FET's, controls, and inductors. These supplies provide $1.2 \mathrm{~V}$ for the FPGA core voltage, $2.5 \mathrm{~V}$ for the FPGA auxiliary supply rail, and a $6.0 \mathrm{~V}$ supply to the four linear regulator 


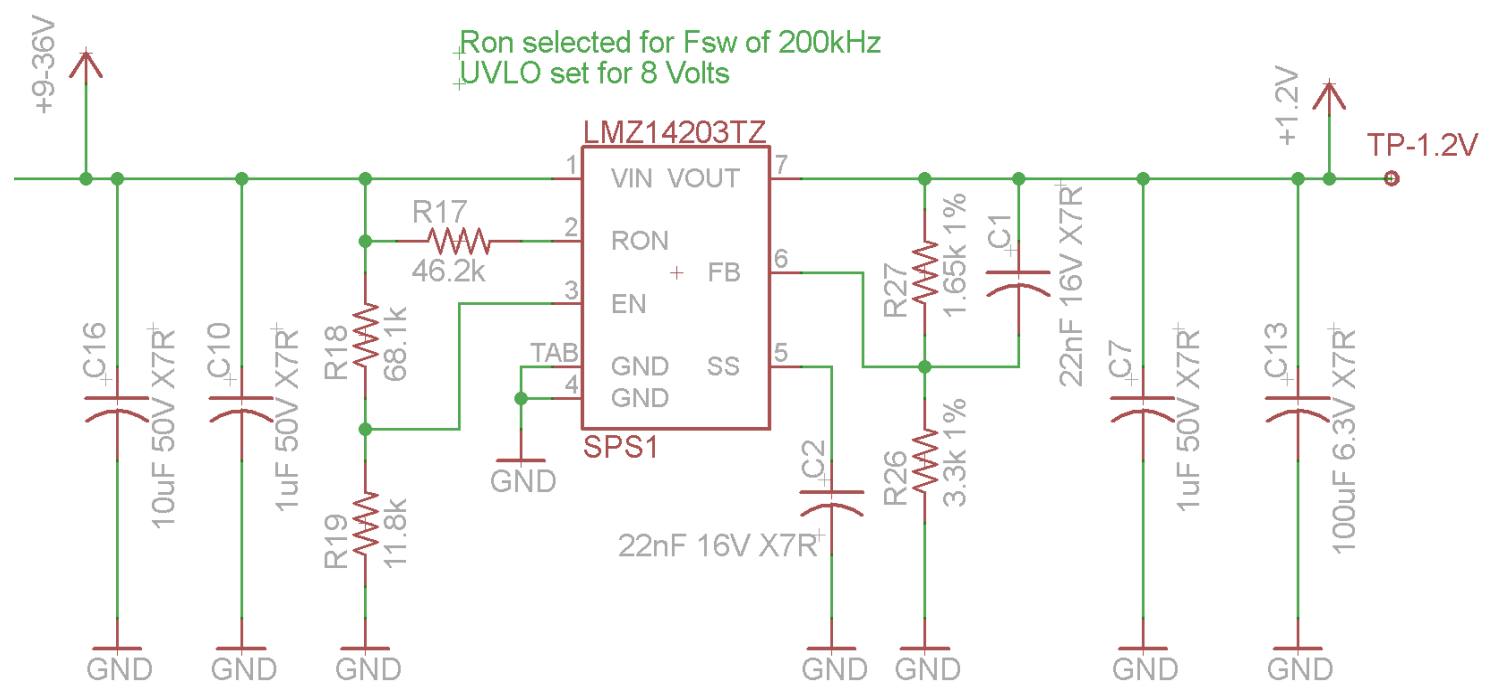

Figure 5.3: Switching power supply, $1.2 \mathrm{~V}$

stages. Figure 5.3 shows the $1.2 \mathrm{~V}$ SPS circuit. The SPS circuits require a minimum input voltage of $6.0 \mathrm{~V}$, so the SPS under-voltage lock out (UVLO) feature is set by R18 and $\mathrm{R} 19$ to turn off the supplies below $8.0 \mathrm{~V}$ at the input. This provides a $2 \mathrm{~V}$ margin between the minimum required input voltage and UVLO. Thus, all supplies will be operational from an input source between $9 \mathrm{~V}$ and $36 \mathrm{~V}$.

The SPS output voltage is selected via R26 and R27, with $\mathrm{C} 1$ added to improve load transient response of the supply. R17 selects the switching frequency of the regulator, with the $1.2 \mathrm{~V}, 2.5 \mathrm{~V}$, and $6.0 \mathrm{~V}$ supplies set to $200 \mathrm{kHz}, 400 \mathrm{kHz}$, and $600 \mathrm{kHz}$ respectively, to ensure continuous mode operation at the expected current consumption. The $1.2 \mathrm{~V}$ and $2.5 \mathrm{~V}$ supplies are designed with $100 \mathrm{uF}$ of bulk capacitance, and the 6.0 V supply with $50 \mathrm{uF}$.

Powered by the 6.0 V SPS, each of the four Linear Technology LT1528Q linear regulators provide a regulated voltage supply for $3.3 \mathrm{~V}$ digital, 3.3 V RF, 5.0 V CAN, 
and 5.0 V RF antenna power circuits. The LT1528Q regulator circuits are shown in Figure 5.4.

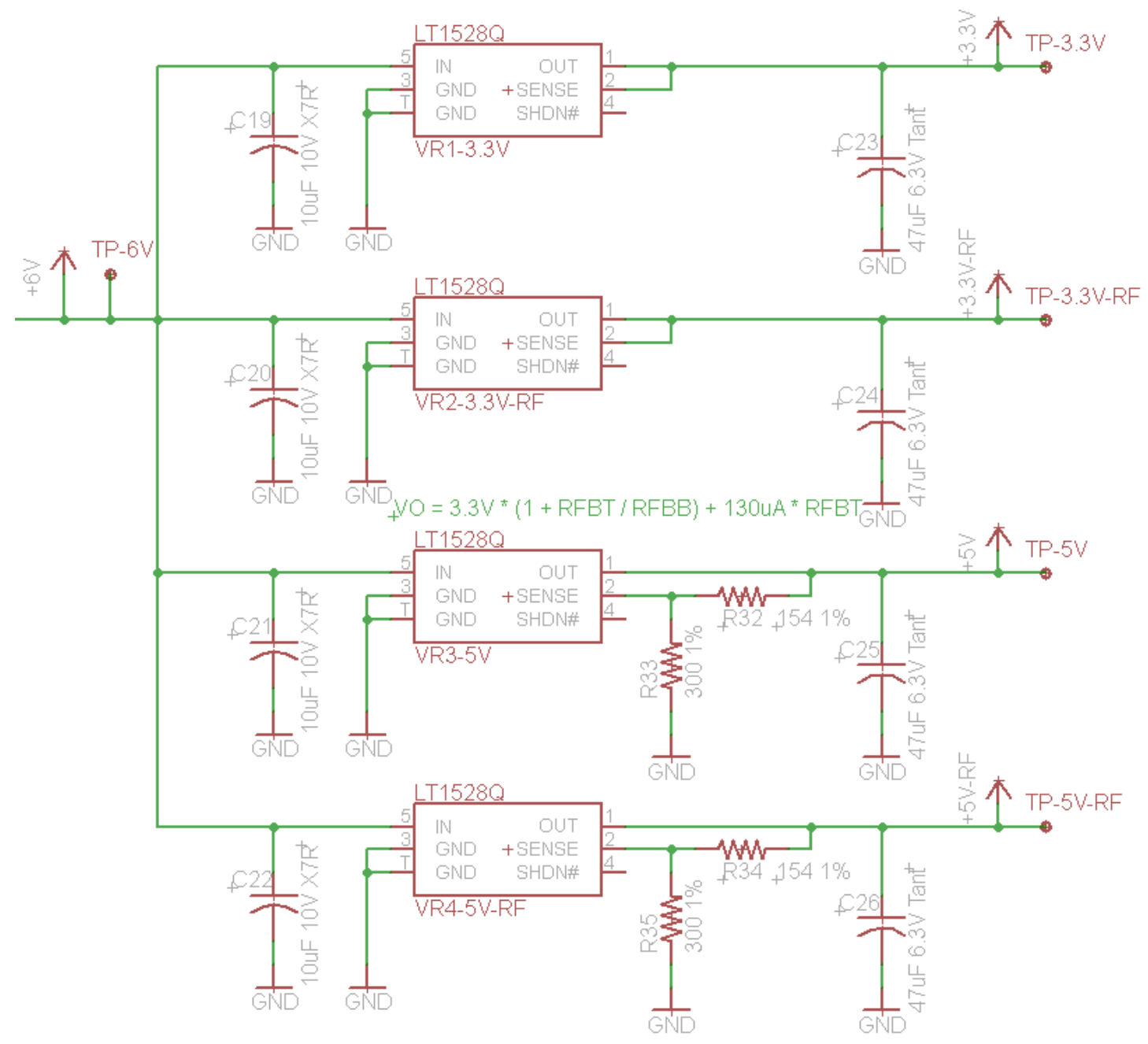

Figure 5.4: Linear voltage regulator circuits

The LT1528Q is a low drop out (LDO) regulator, in that it will supply up to 3 A of current to a circuit with an input voltage that is only $0.6 \mathrm{~V}$ above the output voltage. The LDO regulator creates a 5 V regulated supply sourced from the 6 V SPS. Cascading the SPS and linear regulators minimizes power dissipation from voltage drop across the linear regulators, and thus meets the $2.0 \mathrm{~A}$ max input current rating. Connecting the 
LT1528Q voltage sense pin directly to the output creates a fixed $3.3 \mathrm{~V}$ supply.

Connecting the voltage sense to the output pin via a voltage divider (R32 / R33 and R34 / R35) raises the output voltage, in this case creating 5.0 V supplies. Each of the linear regulators are designed with $10 \mathrm{uF}$ input capacitance and $47 \mathrm{uF}$ output bulk capacitance.

Figure 5.5 shows power distribution paths on the receiver PCB.

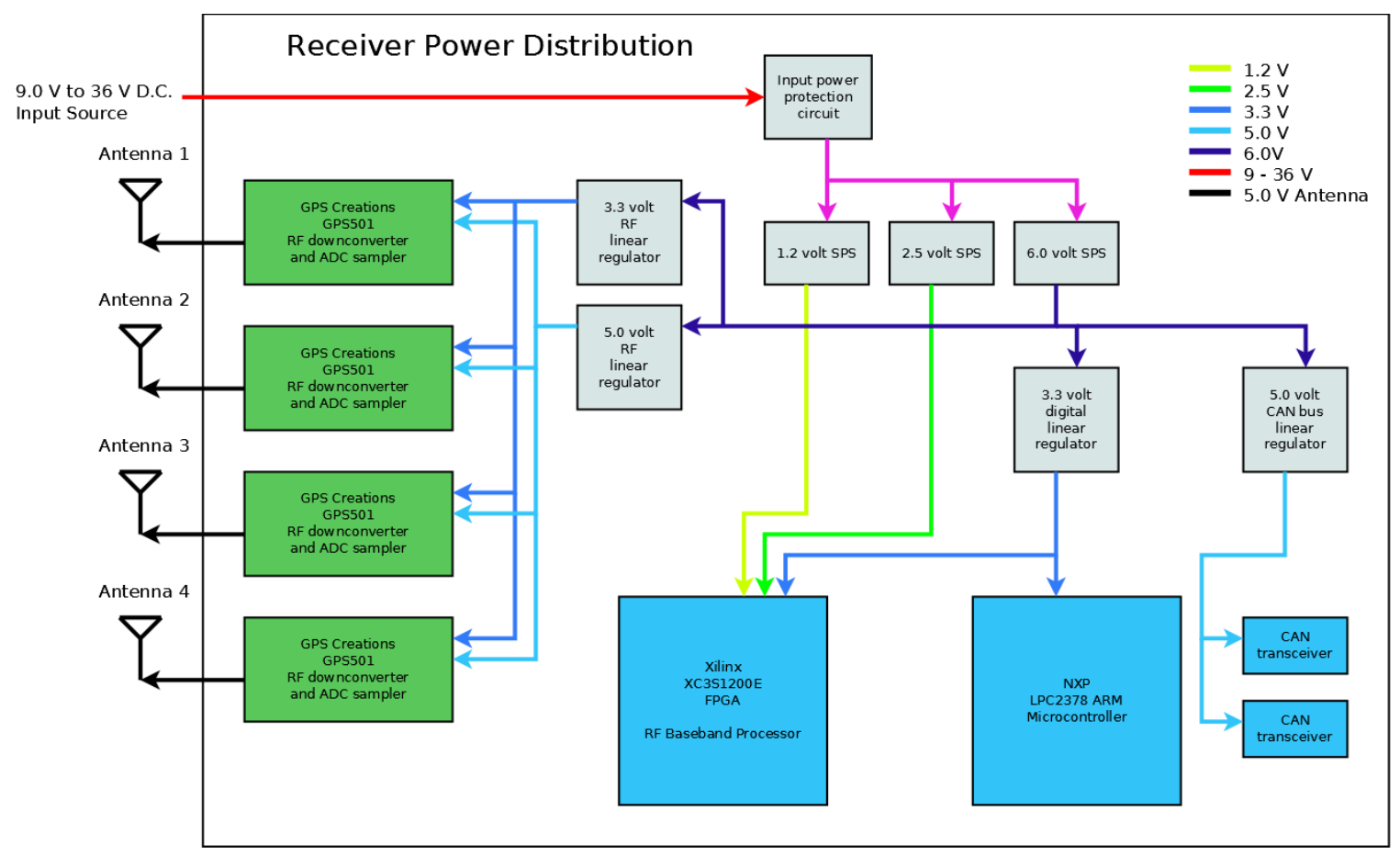

Figure 5.5: Receiver power distribution for all supply voltages 


\subsubsection{ARM processor system}

The microcontroller employed in this design is an NXP Semiconductors LPC2378 ARM7TDMI-S device. The LPC2378 was selected because it is a mature design with few processor errata, it has strong low-cost support hardware, it is supported by a stable open-source tool chain for development, and it requires only a basic PCB layout. The processor uses a 32 bit RISC core based on the ARMv4 instruction set architecture. It contains $512 \mathrm{KiB}$ flash memory, $32 \mathrm{KiB}$ on-chip RAM, and runs at $72 \mathrm{MHz}$ core clock speed. At $72 \mathrm{MHz}$, maximum fast interrupt request (FIQ) latency is less than 19 cycles, which meets the $500 \mathrm{~ns}$ interrupt latency requirement using a $72 \mathrm{MHz}$ core clock speed. Processor peripherals used in this project include general purpose IO (GPIO), a controller area network (CAN) bus, a serial peripheral interface (SPI) bus, and a JTAG interface for processor programming and debugging. The flash space, RAM available and peripheral set may be larger than required; but for an experimental platform, the extra processing resources make sense.

Power to the ARM processor is a single $3.3 \mathrm{~V}$ supply. The $3.3 \mathrm{~V}$ supply is provided to all processor supply pins from the $3.3 \mathrm{~V}$ linear regulator, with $0.1 \mathrm{uF}$ bypass capacitors at all pins. The linear regulated supply is used (opposed to the switching supplies) to provide a clean voltage supply for stability of on-chip PLL circuits as well as low noise for on-chip analog to digital converters.

The processor clock source is provided by a $12 \mathrm{MHz}$ crystal, which is part of the processor's internal clock generation module. A second crystal, at $32.768 \mathrm{kHz}$, is used to drive the internal real time clock (RTC) used for general timekeeping. This clock 
frequency plan follows the reference designs available from NXP, and are used here because they provide the maximum core clock speed of $72 \mathrm{MHz}$.

\subsubsection{FPGA baseband processor}

The receiver uses a Xilinx XC3S1200E Spartan 3E FPGA in a 256 ball BGA package to perform parallel baseband processing. The FPGA employs 1.2 million logic gates, or 19,500 logic cells and over 500,000 bits of block RAM. This is a fairly large FPGA for the Spartan 3E family, and was chosen to provide the most FPGA space for baseband processor experimentation. Larger FPGA's in this family require use of a package with more pins, whereas the 256 ball BGA package is already challenging to layout with only four PCB layers. Therefore, the XC3S1200E is a good compromise of available gates, PCB layout concerns, and power supplies required.

The FPGA is configured as a baseband processor by an automatic transfer of the configuration bits from an XCF04S configuration PROM. When power is applied, the FPGA initiates a data read from the PROM, using the data to configure the FPGA as designed by the user. The configuration PROM and FPGA share a daisy-chained JTAG port for FPGA/PROM programming using a PC and a JTAG adapter cable.

The power supplied to the FPGA includes a $1.2 \mathrm{~V}$ supply to the 'internal', or VCCINT rail in the FPGA core, $2.5 \mathrm{~V}$ to the auxiliary VCCAUX rail, and $3.3 \mathrm{~V}$ was chosen for the VCCO rail for IO pins that interface with the rest of the receiver at $3.3 \mathrm{~V}$.

The FPGA is clocked from a 40MHz reference supplied by the RF front end. This maintains a synchronous design from the RF ADC's through the baseband processor. An additional oscillator pad is connected to the FPGA as a high speed clock source in case it 
is needed, though the $40 \mathrm{MHz}$ oscillator is sufficient for all of the FPGA needs by using the internal clock PLL circuits.

Figure 5.6 details the FPGA and ARM processor interfaces.

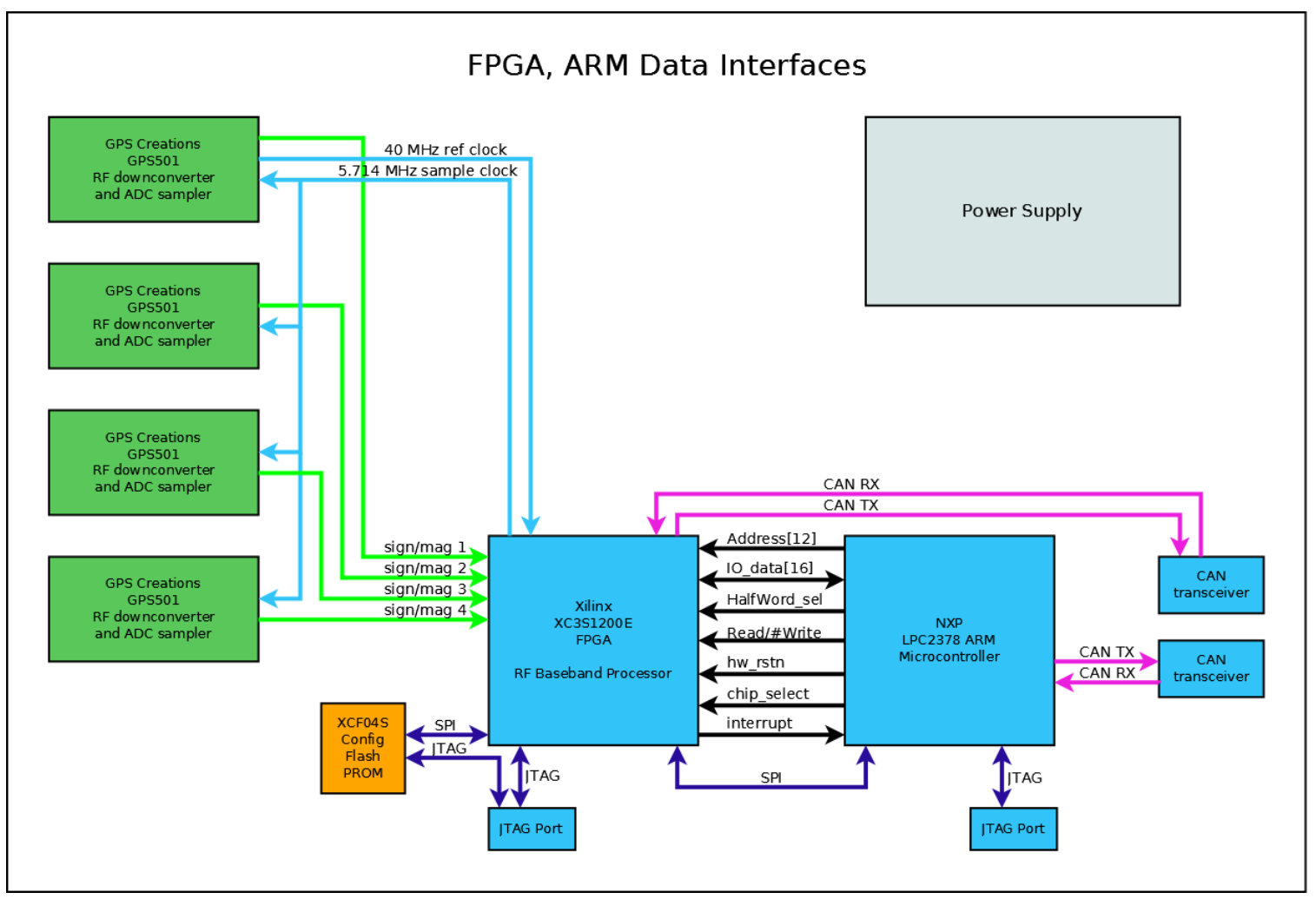

Figure 5.6: Receiver internal data interfaces

The FPGA is interfaced to the ARM processor via two independent buses. One bus uses a serial peripheral interconnect (SPI) interface. This bus uses four wires between the ARM processor (master) and the FPGA (slave). The signals on the wires are SSEL slave select (enabled low), SCK - serial clock, MOSI - master data out, slave data in, and MISO - master data in, slave data out. The SPI ARM-FPGA interface is provided as a secondary data interface, but since the parallel bus has proven sufficient to handle all data transfer, the SPI bus is not used by the processor software or FPGA baseband processor. 
As shown in Figure 5.6, the primary data bus connecting the ARM processor and FPGA is a parallel bus. This bus uses 12 address signals, 16 bi-directional data signals, chip_select (active low), Read/\#Write, and a HalfWord_sel. The bus is capable of data transfers up to 32 bits, which are done 16 bits at a time. For the parallel bus, the ARM processor always acts as the master, and thus controls the bus activity. A read data transfer diagram is shown in Figure 5.7.

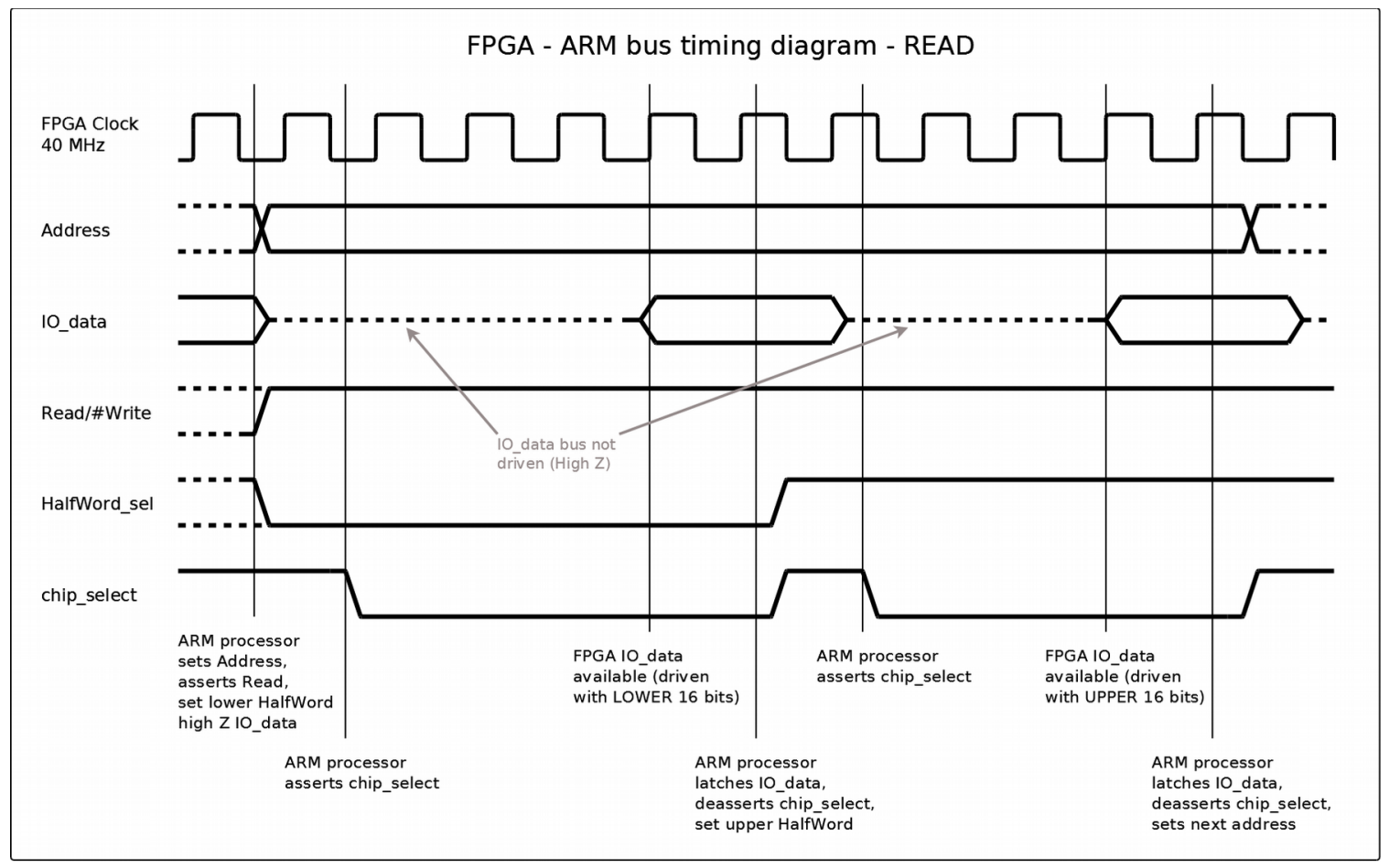

Figure 5.7: FPGA - ARM read cycle timing diagram

A data transfer begins with the master (ARM) setting the address lines for the data to be transferred, setting the ARM data bus direction, setting the Read/\#Write to read (high) for the FPGA data bus direction, and finally asserting the chip_select line for three FPGA clock cycles. Once the transfer is complete, the chip_select and Read/\#Write are de-asserted (set high). Write cycles on the bus occur in a similar manner, except that the 
ARM processor drives the IO_data lines during the cycle, and the Read/\#Write line is set to write (low). 32 bit data words can be exchanged on the parallel bus at a data rate of approximately $3.5 \mathrm{M}$ transfers/second. To support the expected number of correlators, fewer than 200,000 transfers/second are expected, and as few as 12,000 transfers/second under nominal conditions of 24 correlators, 9 transfers/correlator, and every 0.020 seconds.

\subsubsection{RF section}

RF downconversion, RF L1 band selection and filtering, automatic gain control (AGC), and analog to digital conversion (ADC) of the RF signal is performed by a GPS Creations GPS501 L1 band downconverters. The RF section consists of a PCB with attached components and an interface connector. The receiver uses four GPS501 RF sections, one per antenna. The internal frequency plan, selection filters, and block diagram for the GPS501 is shown in Figure 5.8.

The GPS501 receives dedicated power from the 3.3V-RF supply rail, and antenna power from the 5.0V-RF supply rail. The GPS501 submodules are interfaced to the baseband processor FPGA through a single connector interface for each submodule with the signals shown in Figure 5.6. 


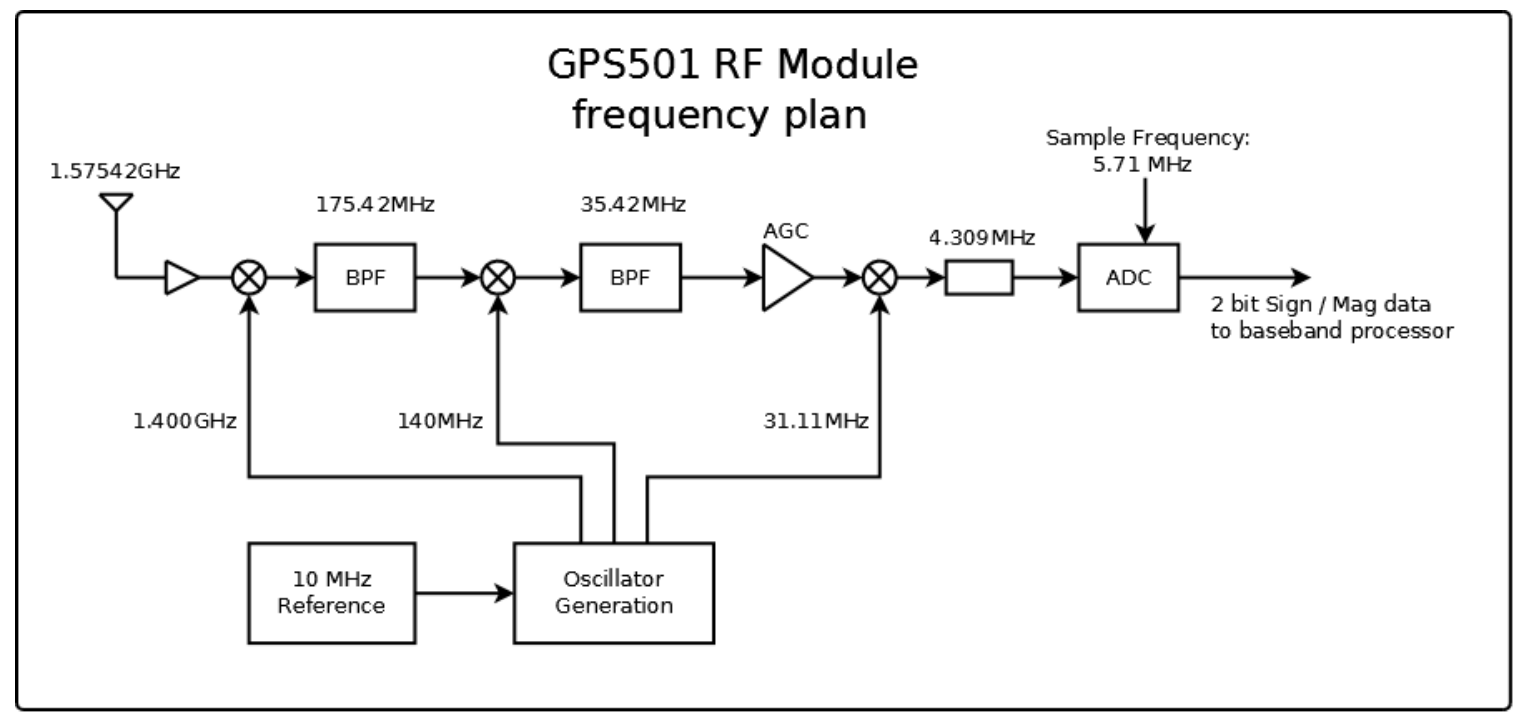

Figure 5.8: GPS501 RF module frequency plan

\subsubsection{CAN communications}

Requirements for communication between the receiver and other embedded systems are met using a CAN bus interface. CAN was chosen because it is a multi-master, self arbitrating, priority enforcing bus design with a 1 Mbit data rate. One CAN transceiver is wired to the ARM processor, and the other is wired to the FPGA. The CAN bus is driven with a Microchip Technology MCP2551 1Mbit CAN transceiver. When the CAN TX input terminal is not being driven (high impedance), the TX input is pulled high by a resistor to de-assert the CAN bus. Further, the MCP2551 is equipped with a timeout function which ignores the TX input and de-asserts the CAN bus if the TX line is low (asserted) for more than 1.25 milliseconds.

The CAN transceivers receive power from a dedicated 5.0V supply rail, bypassed by a $0.1 \mathrm{uF}$ capacitor. The dedicated supply is used to minimize noise transferred by the CAN transceiver into the supply rail from getting into the sensitive analog RF sections or oscillator power supplies. The CAN bus is terminated with a $120 \Omega$ resistor, which will 
give a standard bus impedance of $60 \Omega$ when connected to another device via the CAN bus.

\subsection{HDL design}

The baseband processor was created using Verilog hardware description language (HDL). Like the UNSW team on the Namuru receiver design, this receiver uses a Prompt, Early and Late correlator scheme similar to the (now obsolete) baseband processor chipsets manufactured by Zarlink [3]. Also like the UNSW project, this receiver uses the same GP2015 RF downconverter components on the GPS501 downconverter. The baseband processor's correlator design is given in Figure 5.9.

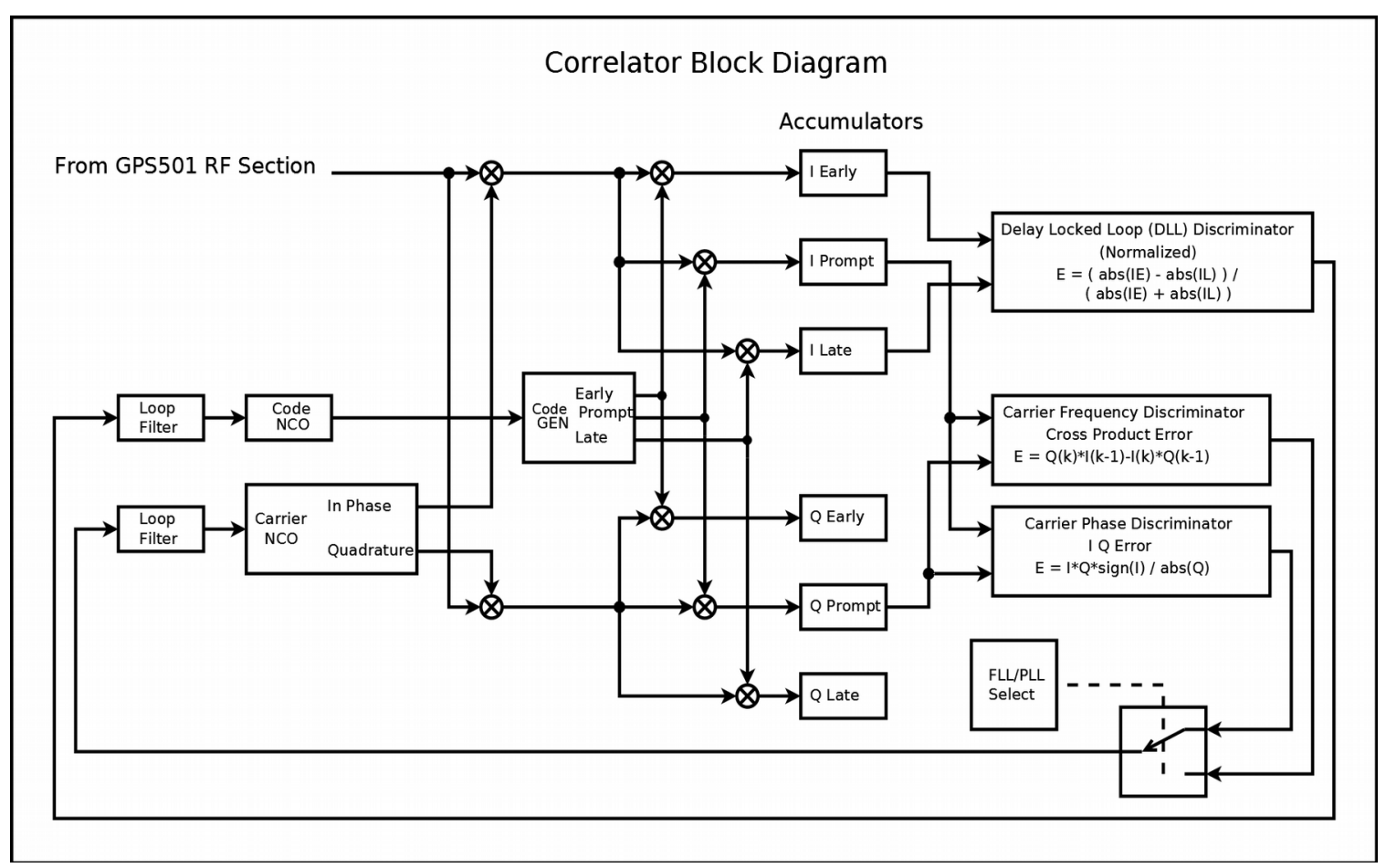

Figure 5.9: Correlator block diagram with tracking loops

In the FPGA baseband processor, the receiver design allows six correlators for each of the four antennas. Each of the correlators track one satellite. To do so, the correlator 
must maintain a local PRN in the code generator (see Figure 5.9) in alignment with the received signal. Multiplying the local PRN signal with the incoming signal from the RF section despreads the signal transmitted from the satellite being tracked. Further, the correlator contains the final local oscillator and mixer which brings the carrier frequency down to baseband, removing the carrier from the received signal. In baseband, the only remaining signal is the satellite navigation message, which toggles the sign of the accumulators inside the correlator. The navigation message comes in at a bit rate of 50 bits/second, so navigation data can be extracted one bit at a time by reading the accumulator values in software.

The PRN code is tracked by minimizing an error signal in the delay locked loop (DLL) discriminator. The discriminator uses the accumulator values inside the correlator to measure the phase error between the local PRN code and the PRN received from the satellite. Once the error is determined in the discriminator, the loop filter adjusts the numerically controlled oscillator (NCO), which drives the frequency of the PRN code generator to keep it aligned with the PRN from the satellite.

Similar to the PRN, the satellite carrier frequency signal is tracked with a phase locked loop (PLL). The PLL uses a carrier phase discriminator which determines the error in the carrier phase using the in-phase and quadrature phase accumulator values. Once the error signal is formed, another loop filter is used to drive the carrier NCO, keeping the local oscillator in phase alignment with the satellite carrier phase.

For the purpose of this receiver, the intent is to compare the carrier phase from multiple correlators, each tracking the same satellite with a different antenna. To do this, 
the sign of the carrier from each correlator is XOR'd, and the result integrated for $1 \mathrm{~ms}$, as shown in Figure 5.10.

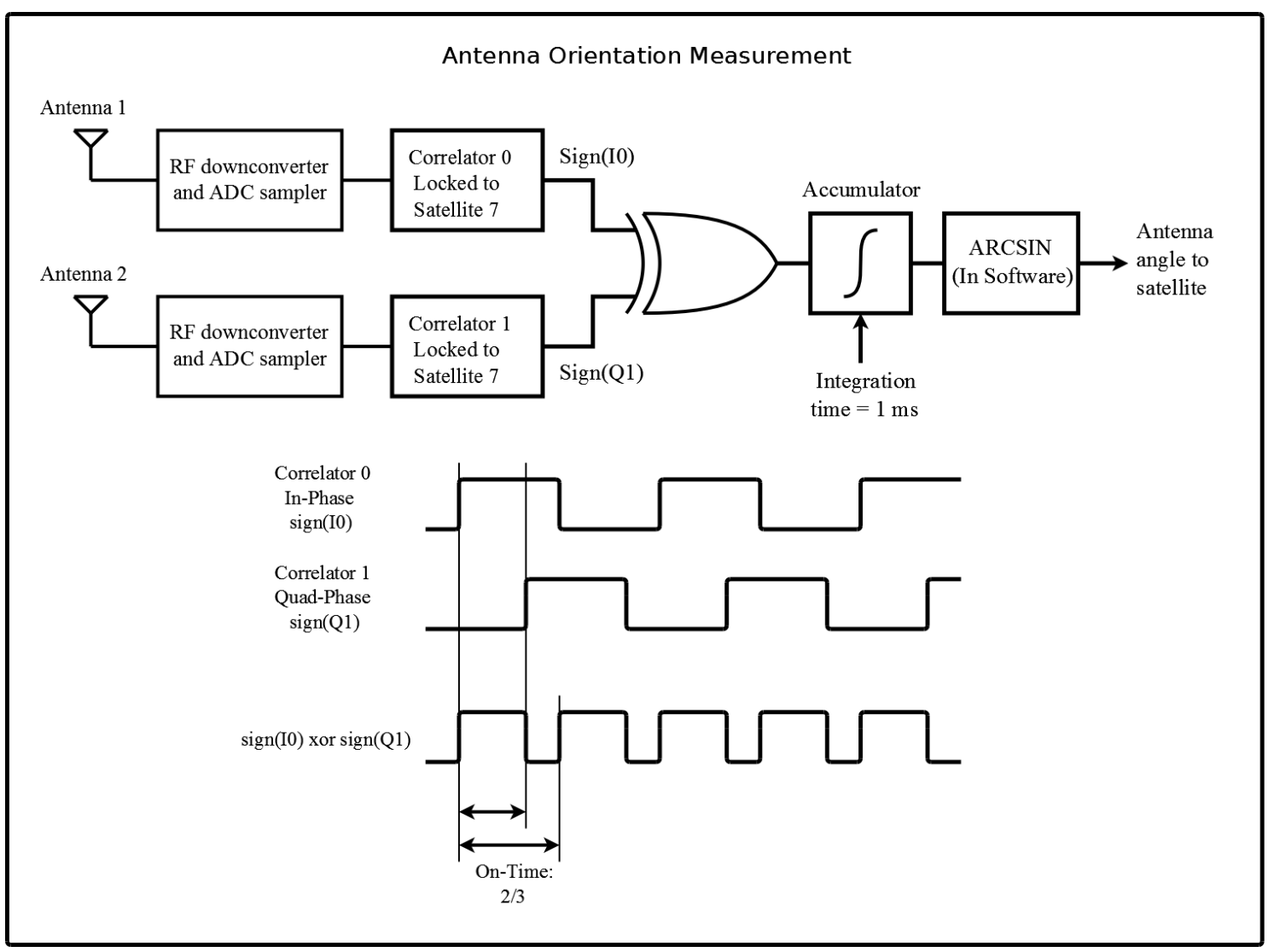

Figure 5.10: Antenna orientation measured from carrier phase

The angle of the two antennas relative to the satellite, $\Theta$, is resolved in software by taking the arcsin of the integration. The combined hardware summation and software computation is given by:

$$
\theta=\sin ^{-1}\left[\left(\frac{2}{N} \sum_{k=1}^{N} \operatorname{sign}(I 0(k)) \text { xor } \operatorname{sign}(Q 1(k))\right)-1\right]
$$




\subsection{Software design}

The software design in this receiver refers to the algorithms, written in $\mathrm{C}$ and assembly language, which are executed on the ARM processor. The software design philosophy for this receiver is to minimize time delay between task execution and hardware data transfer. This is in the interest of rapidly servicing the needs of the baseband processor which could be expanded to many correlators. Further, all GPS processing is performed in one task. Lower priority tasks handle CAN communications and LED indicators. With this simple software layout, nearly any real time operating system (RTOS) could be used, or the software could be run without an operating system using only a one millisecond interrupt and the interrupts from the baseband processor.

The software must maintain a correlator state machine to track the processing of each satellite. For instance, a satellite may progress in state from search; when looking for the signal, to signal found, to PRN track, FLL track, PLL track, and finally the 'identified' state, where satellite position and timing information has been collected and the satellite has been positively identified from the data it is transmitting. Satellite signals can also be lost due to a decrease in signal strength, antenna motion, or movement of the satellite out of view as it orbits the planet. When a satellite is lost, the correlator state machine moves back to a search state; or, if the satellite is no longer in view from the receiver position, a new satellite is selected and the correlator state machine starts over with a search.

To detect and track a satellite signal, the software must perform a search for each satellite with each correlator. The satellite search algorithm sweeps the PRN code 
sequence one phase at a time (of 1023 phases) through the complete PRN code sequence. Each phase is held for $3 \mathrm{~ms}$ in an attempt to find the correct alignment with the satellite PRN. If no signal is found, the carrier oscillator frequency is changed by $250 \mathrm{~Hz}$, and the PRN sweeps are repeated. This process is slow, and could take over 5 minutes to search all PRN phases and carrier frequencies. There are faster search methods, some of which have been described in a thesis or chapter of a book [5]. For this project as a research platform, manual control of the search frequency is possible via the CAN signal viewer (CSV) so that the operator can assist the search algorithm by manually tuning frequencies closer to the expected carrier frequency.

The software also performs the DLL, FLL and PLL loop filter functionality which assists the baseband processor in tracking each satellite. A general control transfer function for a second order loop filter is:

$$
C(s)=\frac{\left(s+\tau_{1}\right)}{s \tau_{2}}
$$

To realize this transfer function in software written in C, a bilinear z transform [6] is used to produce the following difference equation:

$$
Y(k)=Y(k-1)+C_{1} u(k)+C_{2} u(k-1)
$$

The constants $\mathrm{C} 1$ and $\mathrm{C} 2$ are determined by the loop filter sample rate, desired DLL/FLL/PLL tracking bandwidth, and control loop damping ratio. The output of the difference equation, $\mathrm{Y}(\mathrm{k})$, provides the input to the numerically controlled oscillator at a given time step, $\mathrm{k}$. $\mathrm{Y}(\mathrm{k}-1)$ represents the previous output from the equation, and $\mathrm{u}(\mathrm{k})$ and $\mathrm{u}(\mathrm{k}-1)$ represent the present and previous inputs to the loop filter, respectively. Recall 
from Figure 5.9 that the inputs to the loop filter are the results from the phase or frequency discriminators in the baseband processor.

Finally, the software is used to read the carrier phase difference information from the FPGA baseband processor and compute the orientation of the antenna constellation as shown in Equation 5.1.

\subsection{CAN signal viewer}

Using the $\mathrm{C}++$ language and an OpenGL API/library, a real-time live data visualization tool called CAN signal view (CSV) was written specifically for this project. The CSV tool allows the user to decode and plot data received from the project's CAN bus. CAN bus data is collected using an IFAK CAN-to-USB adapter manufactured by isCAN, which comes with $\mathrm{USB}$ drivers and a $\mathrm{C} / \mathrm{C}++$ application programming interface (API).

This very basic CSV-IFAK tool allows large amounts of data to be streamed to a computer screen in a continuously scrolling data plot for qualitative analysis. CSV receives key presses from the computer keyboard to zoom the data plot in and out in the $\mathrm{X}$ (time) axis, and to send CAN bus messages to the receiver. Within the receiver, these messages control which satellite is being searched or tracked, and also what satellite Doppler frequency to search. The CSV tool is the primary tool used to monitor and debug the receiver. 


\subsection{Hardware Implementation}

Design of the circuit schematic and printed circuit board (PCB) was done using Eagle PCB software from Cadsoft. Eagle was selected due to its low cost for designing PCB's less than $160 \mathrm{~mm} \times 100 \mathrm{~mm}$. The PCB is a four layer design to reduce cost, using mostly surface mount components to simplify assembly. Component placement was limited to one side of the board to ease assembling and test probing of the board. The GPS501 RF modules, however, are attached to both sides of the board to limit the total PCB size. Figure 5.11 shows the multilayer PCB design from the PCB design tool view.

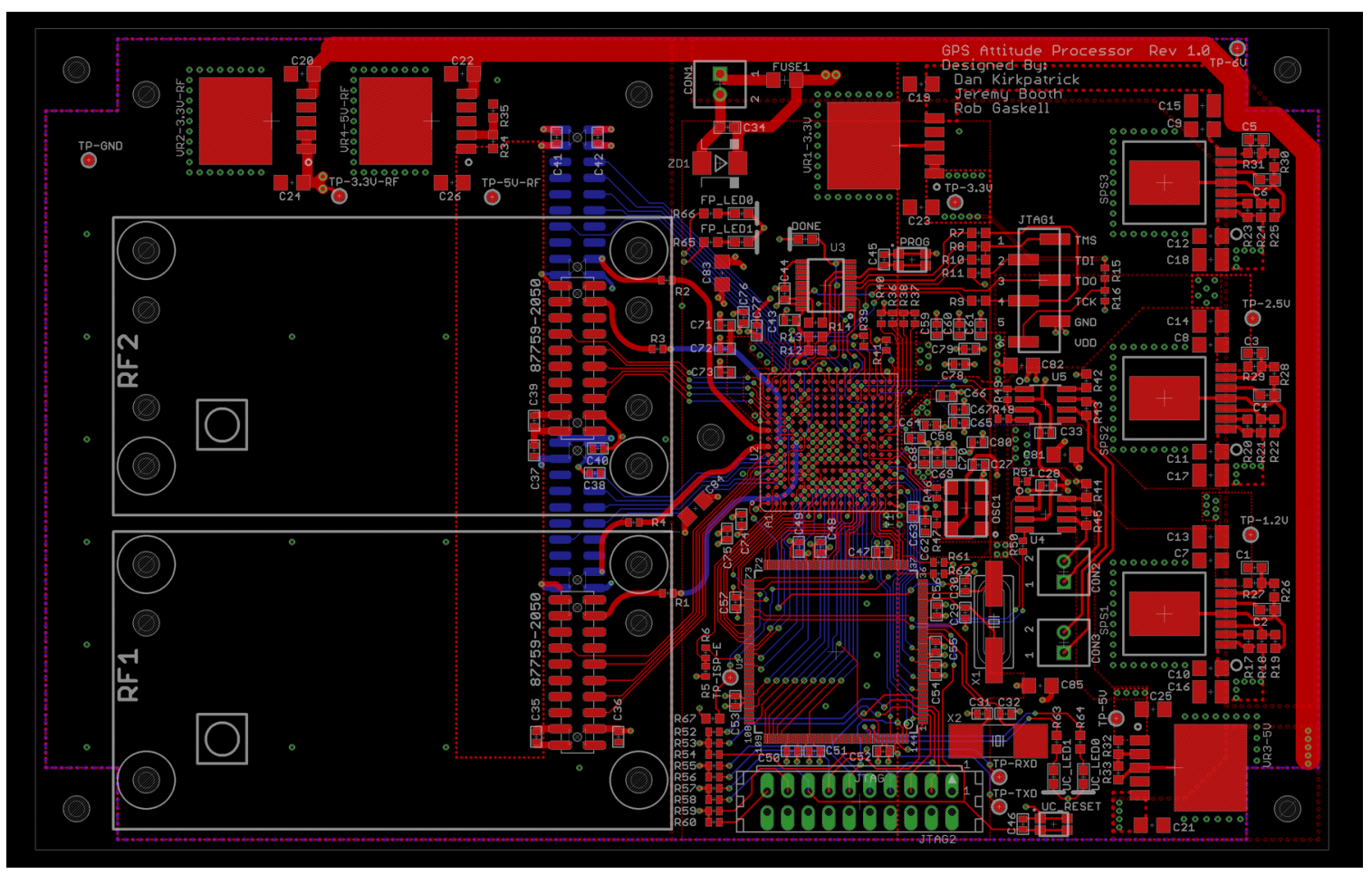

Figure 5.11: Receiver PCB layout

The assembly of the printed circuit board was performed using solder paste and a reflow oven. Hand soldering was used to correct minor problems with solder bridging and to 
install through-hole components. Figure 5.12 shows the complete PCB assembly, denoting the location of the FPGA baseband processor, ARM processor, GPS501 RF sections, and power supply components. Mechanical mounting points are located at the four corners of the PCB and in the center, less than $10 \mathrm{~cm}$ apart to meet mechanical vibration requirements. Screw terminals are provided for power and CAN communication connections, and also meet mechanical requirements.

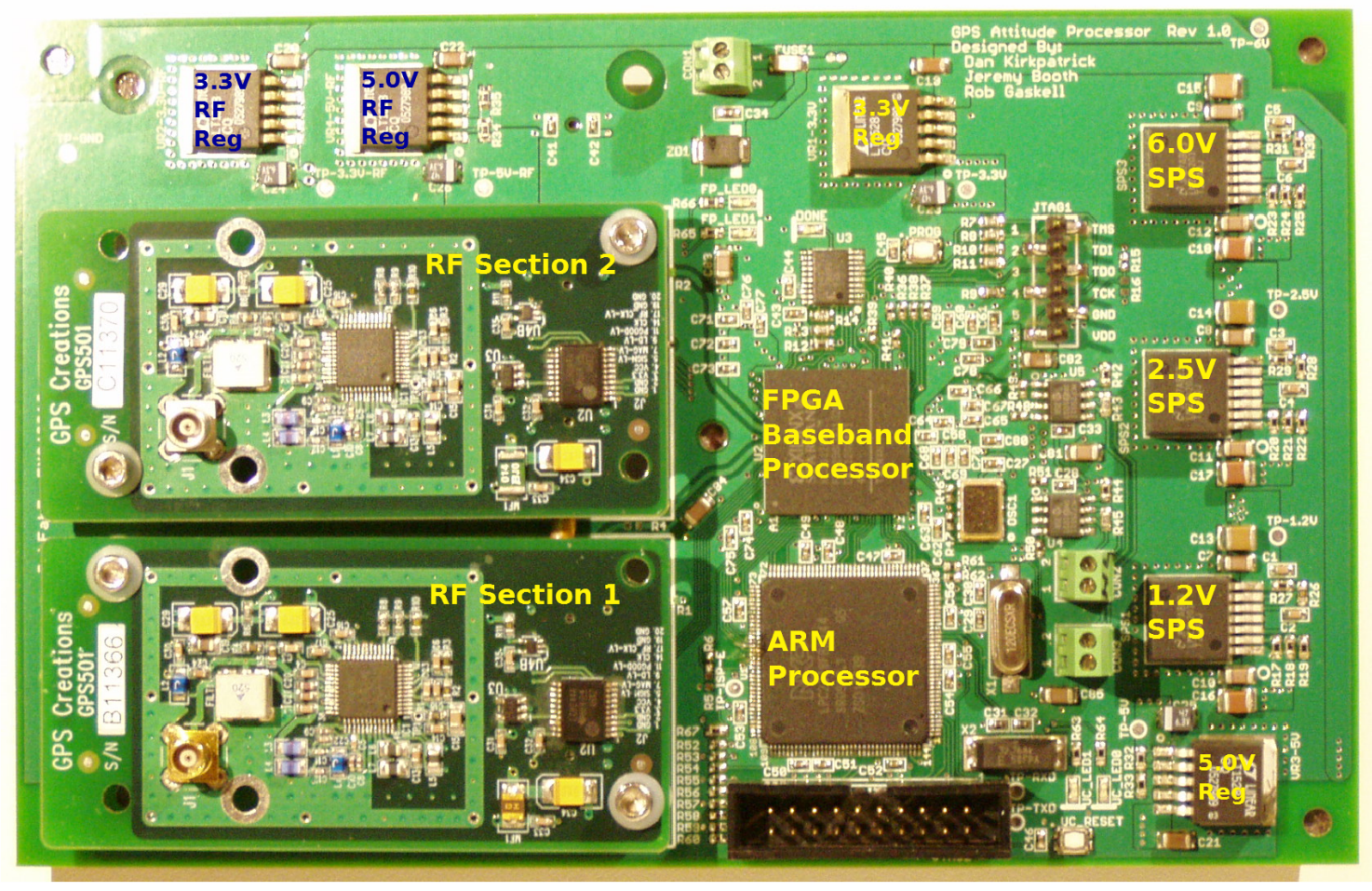

Figure 5.12: PCB assembly with components in place 


\section{Chapter 6 Results}

\subsection{General results}

Power supplies were tested with a nominal $12 \mathrm{~V}$ input voltage. Switching power supplies (SPS's) were observed to be within $0.050 \mathrm{~V}$ of their nominal 1.2 and 2.5 settings, with the 6.0 V SPS within $0.090 \mathrm{~V}$ of its nominal setting. The four linear voltage regulators were also found to be within $0.050 \mathrm{~V}$ of their nominal $3.3 \mathrm{~V}$ and $5.0 \mathrm{~V}$ settings after some component changes for incorrectly sized output capacitors.

Switching power supplies became active above a receiver input voltage of $8.1 \mathrm{~V}$, verifying that the $9.0 \mathrm{~V}$ lower input tolerance has been met with some margin. The maximum input voltage of $36 \mathrm{~V}$ was applied, and all regulators performed as expected. Peak current consumption was $0.260 \mathrm{~A}$ at $8.5 \mathrm{~V}$, and less than $0.080 \mathrm{~A}$ at $36 \mathrm{~V}$. Reversevoltage protection was only measured up to $0.500 \mathrm{~A}$ at $-0.8 \mathrm{~V}$, and voltages over $36 \mathrm{~V}$ were not tested, both due to the destructive nature of the tests. Antenna supply current was verified at each RF section using a $50 \Omega$ load resistor.

An initial configuration was programmed into the FPGA to confirm that the JTAG, PROM, and oscillators worked as intended. This initial configuration initialized all IO pins and flashed LED's connected to the FPGA upon completion. The ARM processor was programmed to flash LED's wired to the processor, as well as to transmit messages over the CAN bus. A simple parallel bus protocol was then programmed to test the FPGA and processor inter-communication. All worked as expected. 
To test the functionality of the hardware platform, software and Verilog was written for the FPGA baseband processor, ARM processor, and CSV monitoring program. The test software was used to verify that the hardware was functioning correctly and that the receiver was capable of tracking satellites as intended. Further, some metrics were needed to verify the resource use in the FPGA. The Table 6 shows the number of lines of software and Verilog written to test four correlators. The correlators are used to track two satellites on two antennas with carrier phase measurements between the antennas.

Table 6: Lines of code written for receiver

\begin{tabular}{|c|c|c|c|}
\hline Device & File & Number of Files & Lines \\
\hline CSV (Windows PC) & $\mathrm{C}++\left({ }^{*} . \mathrm{h},{ }^{*} . \mathrm{cpp}\right)$ & 6 & 1961 \\
\hline FPGA baseband & Verilog $\left({ }^{*} . \mathrm{v}\right)$ & 19 & 3732 \\
\hline ARM processor (all) & $\mathrm{C}, \operatorname{asm}(* . s, * . c, * . h)$ & 40 & 9524 \\
\hline ARM (GPS Only) & $\mathrm{C}(* . c)$ & 2 & 1657 \\
\hline
\end{tabular}

The important metric is the code used to process baseband data, labeled GPS-only in Table 6. The GPS-only ARM code and the FPGA baseband code determine the number and structure of the correlators and phase comparison behavior. The GPS-only ARM and FPGA processing code is less than 6000 lines. For this verification test, the FPGA resource use was only $6 \%$ for both LUTS and slice flip flops, so it is reasonable to assume that with a $6 x$ increase in the number of correlators (to 24 total), scaling linearly would use $36 \%$ of the FPGA at best, and worst case would remain below $50 \%$. This gives margin for expansion, multipath mitigation, and experimentation with other baseband concepts. The software coding style makes use of arrays of structures in the ARM processor, and for-loop iterators in Verilog. Correlators are added to the platform 
by increasing the correlator array counts, adding initialization parameters, and then programming a destination for the new data in the ARM processor.

\subsection{Satellite acquisition results}

For satellite acquisition testing, two correlators were programmed into the FPGA. The LPC2378 processor was programmed to sweep through each pseudo random number (PRN) code phase three times at each satellite Doppler frequency. When a signal was

found, the correlator state machine moved to a pull-in state, during which the delay locked loop (DLL) tracked the PRN for a short time to allow the loop to stabilize. Next, the correlator state machine transitioned to the frequency locked loop (FLL) state, where the correlator loop filter brought the carrier frequency error to near zero. Figure 6.1 shows the CAN signal view (CSV) data as the state machine transitioned toward a FLL satellite lock. 


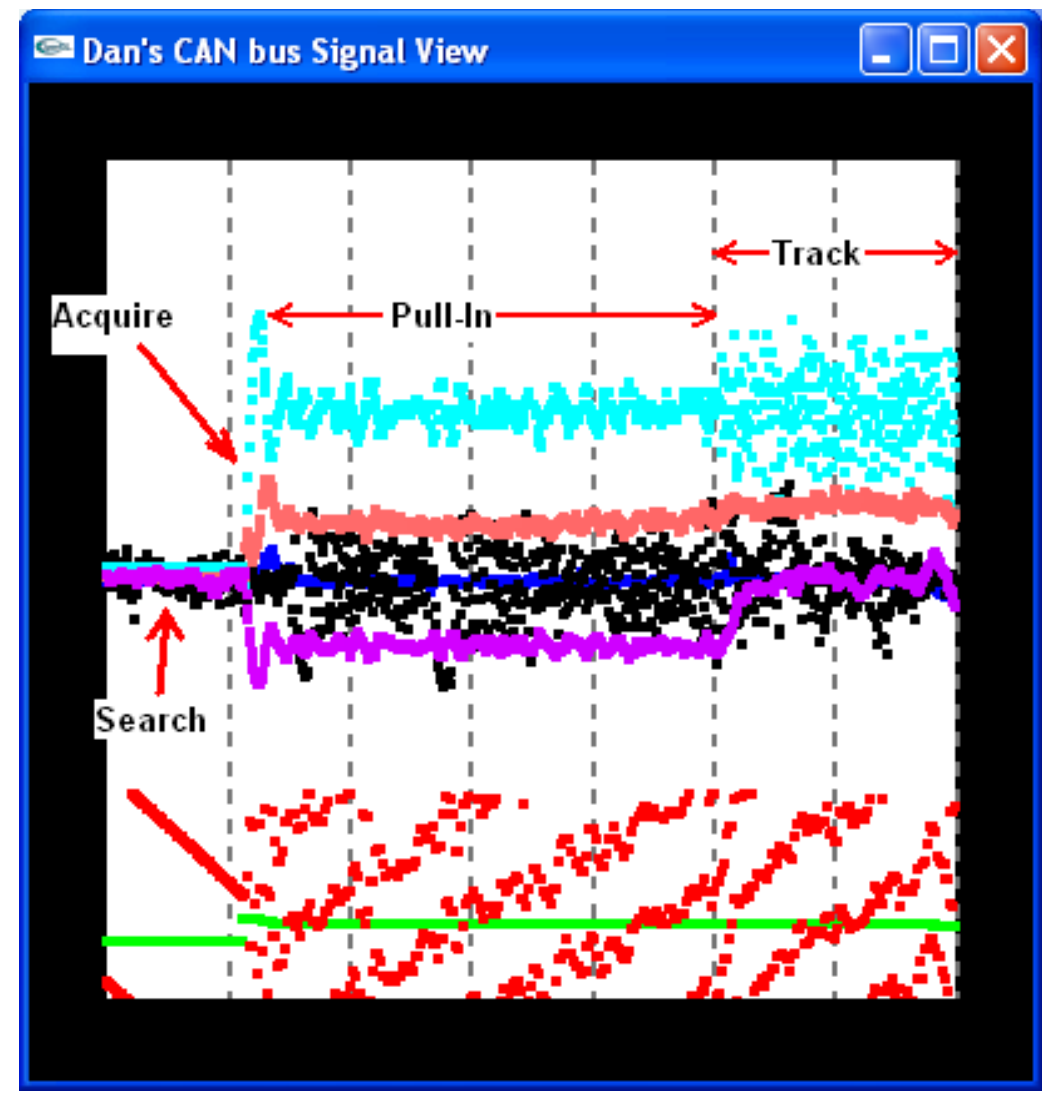

Figure 6.1: CAN signal view of satellite acquisition and DLL/FLL tracking

\subsection{Tracking performance}

After two seconds of tracking the carrier using the FLL and PRN DLL, the state machine transitioned to the phase locked loop (PLL) as shown in Figure 6.2. 


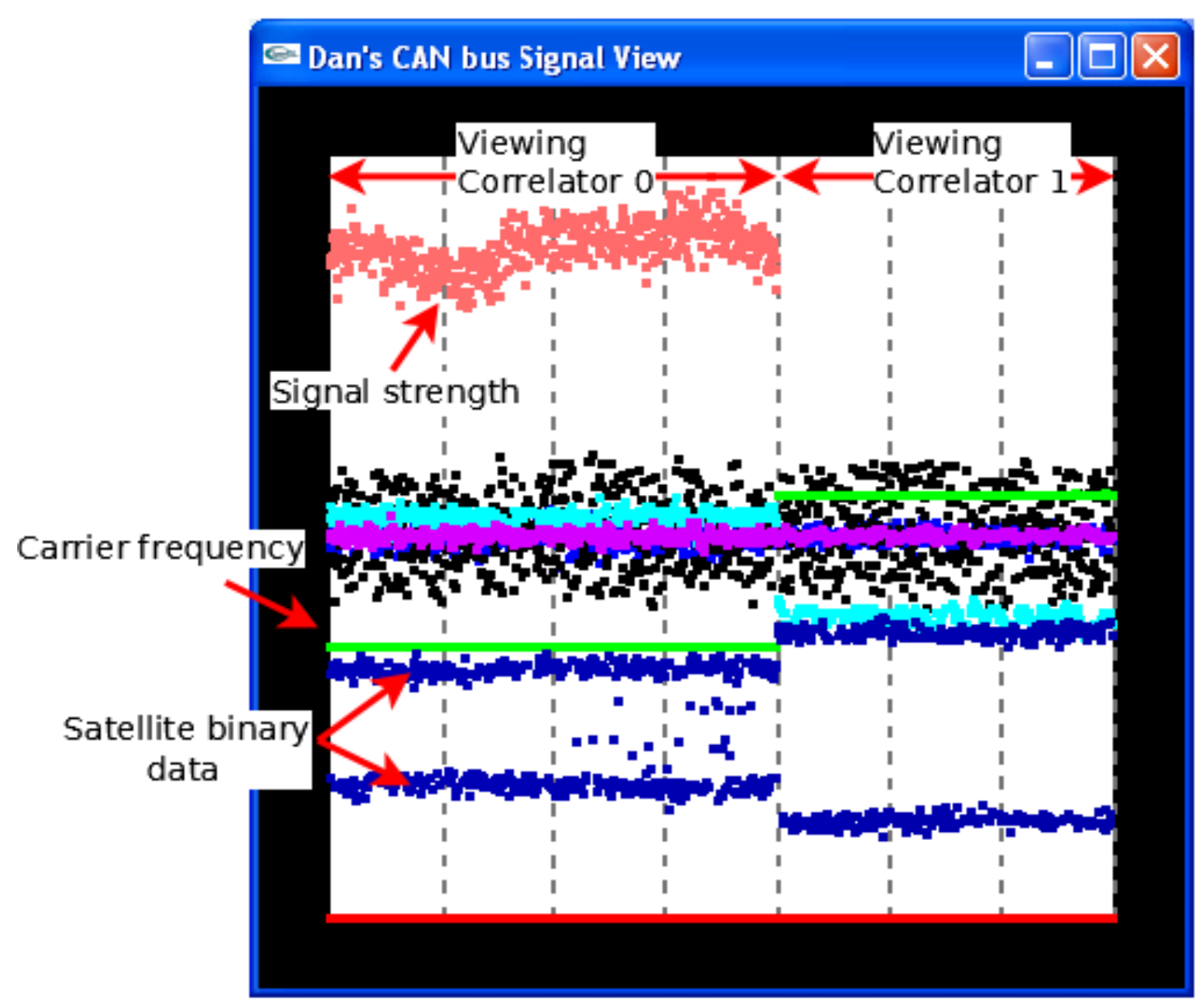

Figure 6.2: CAN signal view of correlators 0 and 1 in PLL track state

In the PLL state, the satellite signal was tracked with the local numerically controlled oscillator (NCO) based carrier, which was phase locked to the satellite carrier. In this state, satellite data was available from the accumulators, and the carrier was available for phase comparison with other correlators. Non-labeled data in Figure 6.2 are debugging signals, which are displayed to observe search and tracking performance. Once a carrier phase lock was obtained, the receiver would typically track the satellite without losing a lock until the satellite passed out of view in its orbit. For this project, satellite positions were confirmed using an internet satellite tracking service:

www.n2yo.com 


\subsection{Carrier phase measurement}

Measurement of the GPS L1 carrier phase relative to multiple antennas is a fundamental concept of this project. To verify that the relative phase measurement can be made with the hardware as designed, a test antenna was constructed using two antennas spaced $1 / 4$ wavelength on a rotating platform as shown in Figure 6.3.

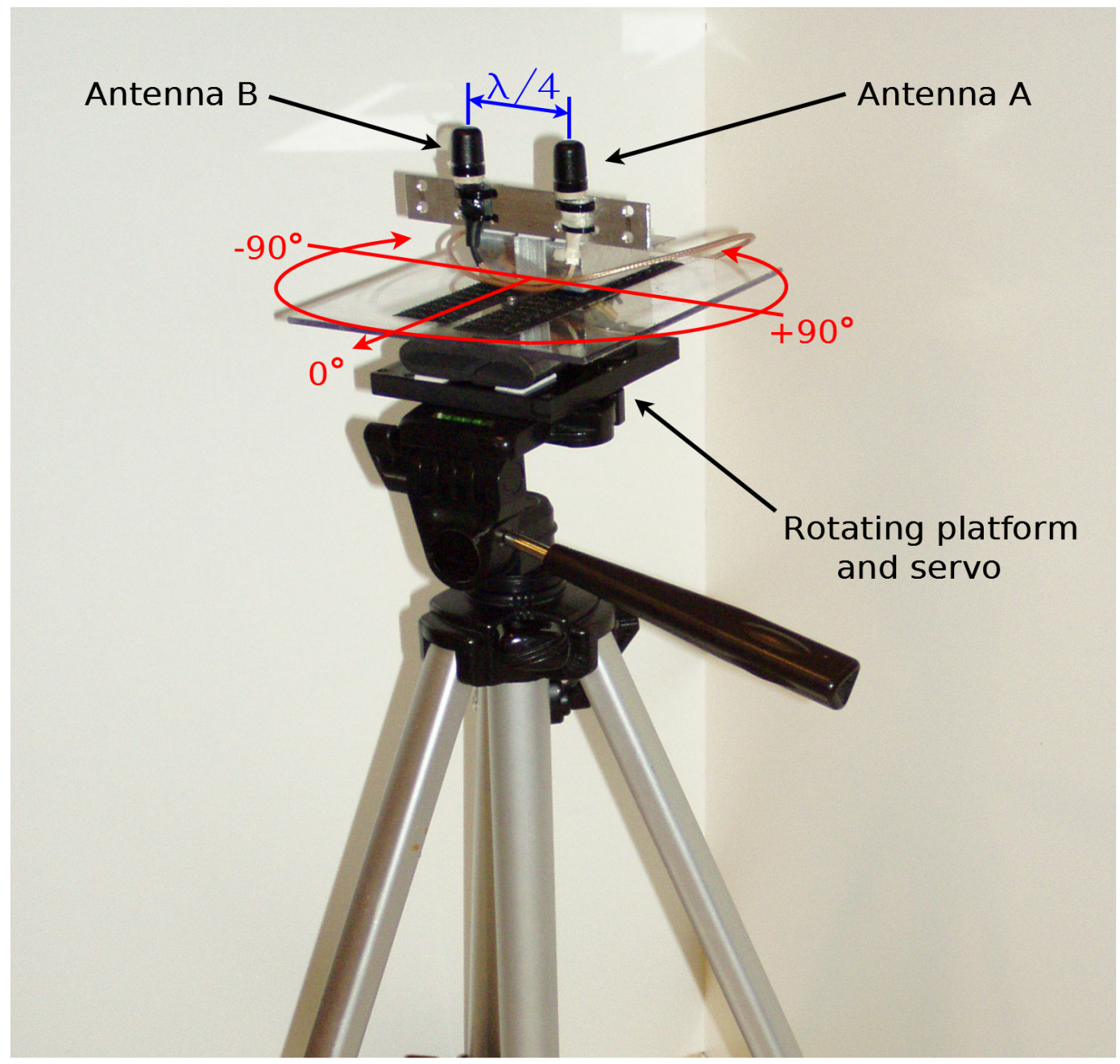

Figure 6.3: Test platform for antenna rotation estimates

The antennas rotated through a range of +90 to -90 degrees using a small radio controlled hobby servo. In the FPGA baseband processor, the local NCO carrier phase 
was measured from antenna $\mathrm{A}$ (master $\mathrm{NCO}$ ) to antenna B (slave $\mathrm{NCO}$ ). To get an accurate measure of the phase relationship between the two oscillators, only the sign of the oscillator (and not the magnitude) need be considered. Phase was computed by taking the XOR of the sign of the two oscillators and integrating that result for a period of time as described in Figure 5.10 and Equation 5.1. Figure 6.4 shows a CSV diagram of the antenna I integration during a slow antenna rotation.

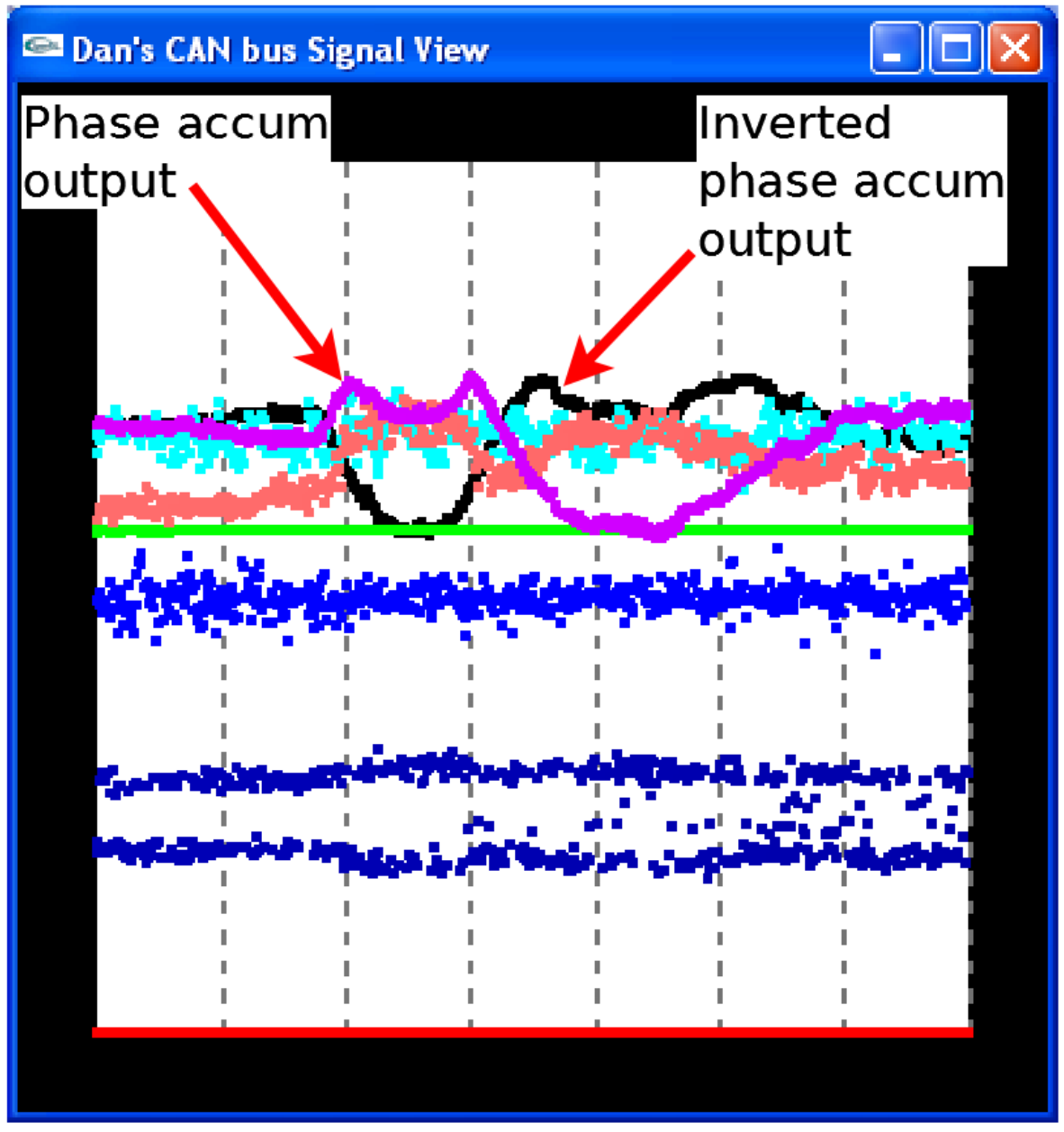

Figure 6.4: CAN signal view graph of I integration during rotation 
During antenna rotation, there is a clear signal from the phase comparison corresponding to the antenna rotation from 0 degrees, to +90 degrees, to -90 degrees, and finally back to zero. This is the raw signal from the phase accumulator, which is the sine of the antenna angle. Despite this test being carried out with a particularly weak signal strength from this particular satellite, the resolved antenna angle was clearly shown in the data. The data in Figure 6.4 also shows an anomaly, in that the shape of the sine wave signal is not continuous and appears to "flip" or reverse direction during the positive phase of rotation. This was caused by a data type error in the CSV program, and not in the receiver or the data from it.

To accommodate a smaller GPS antenna array as needed on a small UAV, an antenna array with a small baseline of $4.76 \mathrm{~cm}$ is used. Small antenna baselines reduce orientation accuracy, but for baselines of $4.76 \mathrm{~cm}$, the integer wavelength orientation ambiguity is not present. The one sigma error expected for a $1 / 4$ wave baseline is $\sim 0.105$ radians, or $\sim 6.0$ degrees [11]. It is the author's opinion that errors in an orientation estimate are acceptable if they are bounded; and that bounded errors in orientation are preferred over a more accurate orientation estimate that is unstable under some conditions. The justifying argument for orientation error tolerance is that flying rotorcraft (helicopters, quadcopters, etc.) must adjust their orientation to maintain flight in the presence of unknown and unpredictable wind. Even if the orientation were exactly known, the flight control system must significantly adjust the orientation to maintain a desired flight condition (such as cruise, descent, or hover). The contention is, for the flight control, a (sufficiently small) error in the orientation estimate is indistinguishable 
from an unpredictable wind or other atmospheric conditions. If the change in orientation necessary to compensate for winds is greater than the absolute orientation sensor error, then robust control is possible.

\subsection{Challenges}

Despite the success of the single axis antenna angle determination, one significant hurdle is left to be resolved before this receiver is ready to measure a full GPS-based attitude solution. That problem is the error caused by multipath interference, as shown in Figure 6.5.

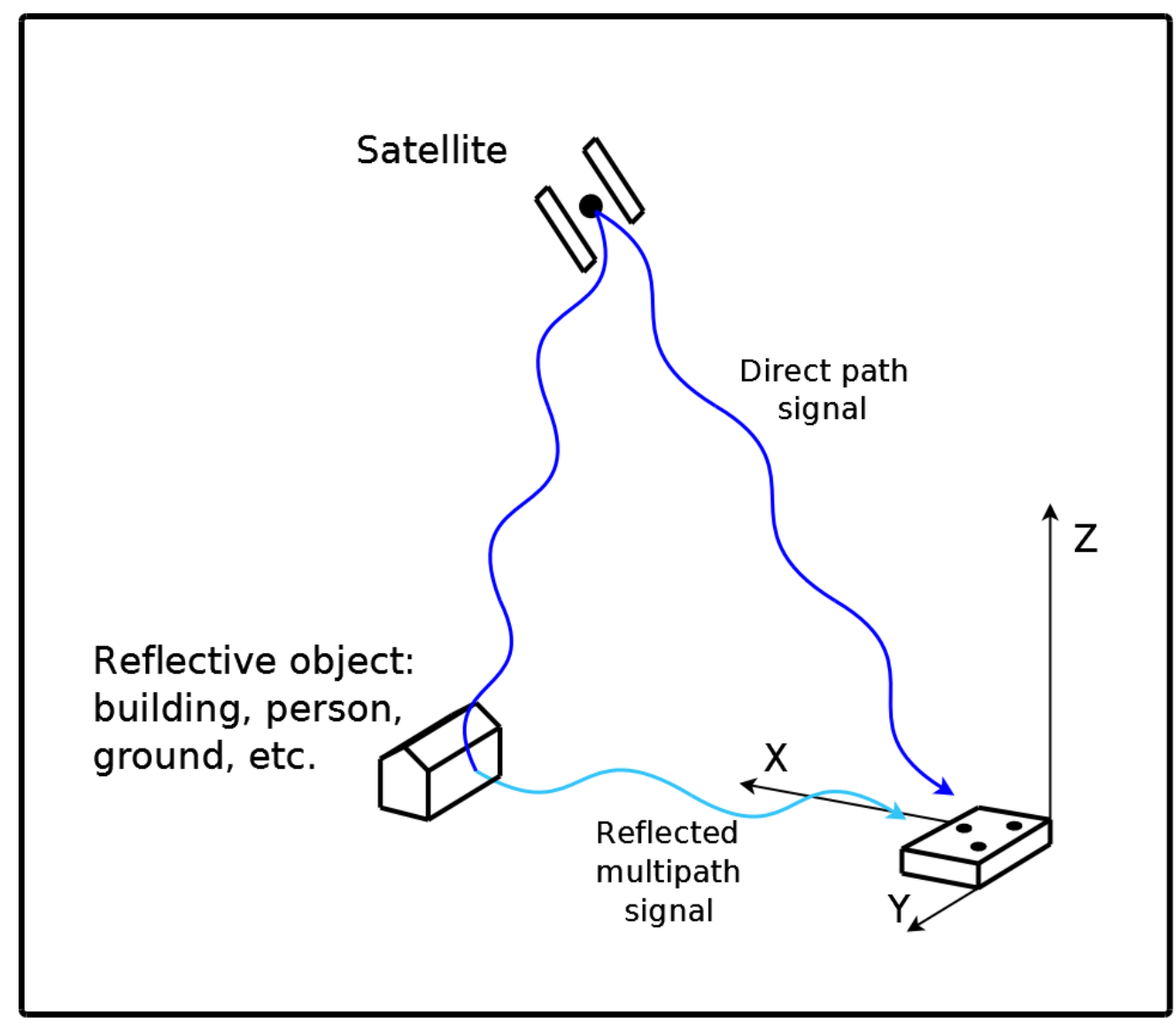

Figure 6.5: Multipath signal interference arriving at antenna array 
Multipath interference refers to the condition whereby the signal from the satellite arrives at the receiver after being reflected off of another object. Sometimes, the multipath signals were received at the antenna by reflection off of a person standing near the antenna. The receiver may track the signal directly from the satellite, or it may track the reflected signal. In some cases, the reflected signal may be the stronger one, because the direct-path signal is blocked by some object. In the presence of multipath interference, the receiver tracking loops would occasionally begin to tracking a multipath signal instead of the direct path signal. When that happened, the estimate of the angle to the satellite would jump to an incorrect value. This happened because based on the carrier phase, the signal "appears" to come from a different direction.

Multipath mitigation techniques are a huge topic of study in the field of global navigation satellite system (GNSS) receivers [5], and they are beyond the scope of this hardware platform project. However, further research into multipath mitigation will be required so that this receiver can be used for orientation measurement [11]. 


\section{Chapter 7 Conclusion}

\subsection{What to do about challenges}

Multipath interference is a significant challenge in this project, and mitigation techniques are exactly the kinds of things which could be studied with this receiver. Reference [5] describes the effects of multipath interference and some of the technology used to mitigate the errors.

One significant method to reduce multipath errors is to use early and late correlators which are close in time delay (close correlator spacing). This may be a complicated solution because it requires changes to the RF sections. The RF bandwidth must be widened as the correlator spacing is narrowed, otherwise the signal to noise ratio will suffer [8]. Despite the need to change the RF sections, close correlator spacing is considered the norm in modern correlator designs due to the effective reduction of multipath signals from greater than $100 \mathrm{~m}$ path differences.

Another effective multipath reducing technique is the use of a gated correlator. Gated correlators concentrate the effect of correlation in the areas near the transitions of the PRN code [9]. This helps the receiver to ignore signals which are delayed from the direct path, and also narrows the autocorrelation function.

\subsection{Future research and study}

There are many other areas to research in a GPS receiver platform. Satellite search algorithms, inertially aided tracking loops, advanced forms of discriminator normalization, clock phase noise evaluation, and multi-constellation receivers are a few. 
All of these topics can be studied with the receiver as built. The first topic for further study will be multipath mitigation, and following that, a full orientation solution will be computed.

Data collection and visualization is another significant area of this project which needed improvement. When dealing with large amounts of noisy data, having ways to plot and evaluate the data is critical. Visualizing live data in terms of time, other data, and numerical text is important and must be done to see data trends and debug coding errors. While CAN signal view is an effective qualitative date viewer, numeric scales, the ability to change vertical scale, logarithmic scales, and signal rewind are needed to complete its usefulness.

Future versions of this receiver could include a processor with hardware floating point support. The original plan had this platform as a sensor only, with the heavy orientation processing done off board on a high performance Intel x 86 based CPU in a PC104+ form factor. Because microcontroller performance has increased since this design commenced, the orientation processing could be done in a modern microcontroller on board the receiver. For example, the NXP LPC2378 used in this design uses a single processor core at $72 \mathrm{MHz}$ and no hardware floating point support. In contrast, the newer NXP LPC4300 can run at $200 \mathrm{MHz}$ with single precision floating point support and two separate processing cores.

Another processing alternative could be the TI RM57x processor, which uses an ARM R5F core at $300 \mathrm{MHz}$ and provides a double precision floating point processor. The processor is designed for safety critical applications, so the cost is higher than many 
others. However, the RM57x provides high performance for a single chip microcontroller.

Finally, there are processor options such as the TI AM335x series at low cost versus performance. These processors provide double precision floating point execution, single precision vector floating point operation, and execution at speeds of $1 \mathrm{GHz}$. The AM335x processors use DDR RAM, off chip flash memory, and complex power control logic. The drawback to moving to processors like the AM335x is the design and layout complexity and noise. The use of DDR RAM requires controlled circuit trace impedance and proper layout to ensure correct signal timing. Incorrect layout can cause data errors and RF noise emissions which would interfere with a GPS receiver. The PCB layout complexity and noise susceptibility for DDR RAM may out weight the speed benefits for this receiver.

\subsection{Summary}

The objective of this work was to provide a receiver hardware platform which could support research on GPS-based attitude determination. The receiver envisioned, designed, built, and tested is able to find and track GPS L1 satellite signals using four RF sections connected to an array of antennas. A GPS baseband processor was written in Verilog for execution on an FPGA. The ARM processor was programmed to search for and track GPS satellites. The receiver can be powered from a wide range of battery voltages, which makes it suitable for portable operation. The receiver is contained on a PCB which is $10 \mathrm{~cm} \times 15.5 \mathrm{~cm}$, and can be attached to and flown on a mid sized RC helicopter for live signal flight testing. The hardware is working, though it is the author's 
opinion that multipath mitigation logic in the FPGA baseband processor is necessary. Without multipath mitigation, the antenna orientation is unusable due to the dramatic orientation errors of 90 degrees or more. Literature suggests that the multipath mitigation problem in this receiver is a solvable one, and therefore this receiver can be effective as a hardware platform to experiment with GPS based attitude determination. 


\section{References:}

[1] Tsai, Chun-Ming. Navigation System for a Helicopter UAV. B.ASc thesis, Simon Fraser University, Burnaby, BC, Canada, August 2004

[2] Greenberg, Andrew. Open Source Software for Commercial Off-The-Shelf GPS Receivers. M.S.CpE thesis, Portland State University, Portland, OR, 2005

[3] Mumford, P.J., Parkinson, K., Dempster, A., "The Namuru Open GNSS Research Receiver," Proceedings of the 19th International Technical Meeting of the Satellite Division of The Institute of Navigation (ION GNSS 2006), Fort Worth, TX, September 2006, pp. 2847-2855.

[4] Cohen, C., McNally, B., Parkinson, B. Flight Tests of Attitude Determination Using GPS Compared Against an Inertial Navigation Unit. Proc. ION National Technical Meeting, San Francisco, CA, Jan 1993.

[5] Grewal, Mohinder S., and Lawrence R. Weill. Global Positioning Systems, Inertial Navigation, and Integration. New York: John Wiley, 2001. Print.

[6] Franklin, Gene F., and J. David Powell. Feedback Control of Dynamic Systems. 4th ed. Upper Saddle River, NJ: Prentice Hall, 2002. Print. 
[7] Tsui, James Bao. Fundamentals of Global Positioning System Receivers: a Software Approach. 2nd ed. New Jersey: John Wiley \& Sons, 2005. Print.

[8] Kiesel, S., Langer, M., Eckert, M., Trommer, G., Discriminator Weighting and Performance of a Deeply Coupled GPS/INS System at low CNO. Proc. IONGNSS 2011, pages 858--867, San Diego, CA, Jan. 2011.

[9] McGraw, Gary A., Braasch, Michael S., GNSS Multipath Mitigation Using Gated and High Resolution Correlator Concepts, Proceedings of the 1999 National Technical Meeting of The Institute of Navigation, San Diego, CA, January 1999, pp. 333-342.

[10] Favor, Michael. Artist Interpretation of GPS satellite, satellite image courtesy of NASA Web. Feb 2006.

[11] Parkinson, Bradford W. Global Positioning System Theory and Applications.

Volume II. Washington, D.C.: American Institute of Aeronautics and Astronautics, 1996. 535. Print.

[12] Incropera, Frank P., and David P. DeWitt. Fundamentals of Heat and Mass Transfer. 4th ed. New York: Wiley, 1996. Print. 